\title{
THE RATE OF CONVERGENCE OF THE WALK ON SPHERES ALGORITHM
}

\author{
ILIA BINDER AND MARK BRAVERMAN
}

\begin{abstract}
In this paper we examine the rate of convergence of one of the standard algorithms for emulating exit probabilities of Brownian motion, the Walk on Spheres (WoS) algorithm. We obtain the complete characterization of the rate of convergence of WoS in terms of the local geometry of a domain.
\end{abstract}

\section{INTRODUCTION}

Brownian Motion (BM) is the most important model of randomized motion in $\mathbb{R}^{d}$. It is the simplest (but, in a sense, generic) example of a continuous diffusion process. BM has found an astonishing number of application to diverse areas of Mathematics and Science, including Biomathematics, Finance, Partial Differential Equations, and Statistical Physics [EKM97, KS98, Maz02, Nel67, Szn98].

Because of the ubiquity of BM, its effective simulation provides a way to efficiently solve a variety of problems, such as computation of Conformal Maps, Tomography, and Stochastic PDEs. One of the main ways in which simulations of BM are used is to study its first hitting probabilities with respect to some stopping conditions. A particularly interesting stopping condition is of hitting the boundary of some topologically open bounded connected domain $\Omega$. For any starting point $x$, the harmonic measure $h_{x}$ on $\partial \Omega$ is given by the hitting point distribution. In many of the BM's applications, it is enough to obtain information about the harmonic measure, more specifically, to efficiently sample from it.

One of the immediate applications of the ability to sample from harmonic measures is solving the Dirichlet problem in $\mathbb{R}^{d}$. The Dirichlet problem on a domain $\Omega \subset \mathbb{R}^{d}$ with continuous boundary condition $f: \partial \Omega \rightarrow \mathbb{R}$ is the problem of finding a $C^{2}$-smooth function $u: \Omega \rightarrow \mathbb{R}$ satisfying

$$
\begin{cases}\Delta u(x)=0 & x \in \Omega \\ u(x)=f(x) & x \in \partial \Omega\end{cases}
$$

In other words, finding a harmonic function $u$ subject to the boundary conditions $f$. By the celebrated Kakutani's Theorem [Kak44, GM04], the value of $u$ at $x \in \Omega$ is exactly the expected value of $f$ with respect to the harmonic measure $h_{x}$ on $\partial \Omega: u(x)=\mathbf{E}_{h_{x}(z)}[f(z)]$.

In the present paper we study the amount of time it takes to sample from the harmonic measure with precision $\varepsilon$ using the Walk on Spheres algorithm - the simplest and most commonly used algorithm for sampling from the harmonic measure.

2000 Mathematics Subject Classification. 60G42, 65C05, 31B25, $31 \mathrm{~B} 05$.

Key words and phrases. Walk on Spheres algorithm, Harmonic measure, Potential Theory.

Both authors were partially supported by their respective NSERC Discovery grant. This research was partially conducted during the period the second author was employed by the Clay Mathematics Institute as a Liftoff Fellow, and as a postdoctroal researcher with Microsoft Research New England.

${ }^{1}$ A preliminary version of this work appeared in the proceedings of the 20th ACM-SIAM Symposium on Discrete Algorithms (SODA09) 
1.1. The Walk on Spheres algorithm. Let us now define the Walk on Spheres algorithm (WoS). We would like to simulate a BM in a given bounded domain $\Omega$ until it gets $\varepsilon$-close to the boundary $\partial \Omega$. Of course one could simulate it using jumps of size $\delta$ in a random direction on each step, but this would require $O\left(1 / \delta^{2}\right)$ steps. Since we must take $\delta=O(\varepsilon)$, this would also mean that the process may take $\Omega\left(1 / \varepsilon^{2}\right)$ steps to converge.

The idea of the WoS algorithm is very simple: we do not care about the path the BM takes, but only about the point at which it hits the boundary. Thus if we are currently at a point $X_{t} \in \Omega$ and we know that

$$
d\left(X_{t}\right):=d\left(X_{t}, \partial \Omega\right) \geq r,
$$

i.e. that $X_{t}$ is at least $r$-away from the boundary, then we can just jump $r / 2$ units in a random direction from $X_{t}$ to a point $X_{t+1}$. To justify the jump we observe that a BM hitting the boundary would have to cross the sphere

$$
S_{t}=\left\{x:\left|x-X_{t}\right|=r / 2\right\}
$$

at some point, and the first crossing location $X_{t+1}$ is distributed uniformly on the sphere. There is nothing special about a jump of $d\left(X_{t}\right) / 2$ and it can be replaced with any $\beta d\left(X_{t}\right)$ where $0<\beta<1$.

Let $\left\{\gamma_{t}\right\}$ be a sequence of i.i.d. random variables each being a vector uniformly distributed on the unit sphere in $\mathbb{R}^{d}$. We could take, for example, $\gamma_{t}=\Gamma_{t}^{d} /\left|\Gamma_{t}^{d}\right|$, where $\Gamma_{n}^{d}$ is a normally distributed $d$-dimensional Gaussian variable. Then, schematically, the Walk on Spheres algorithm can be presented as follows:

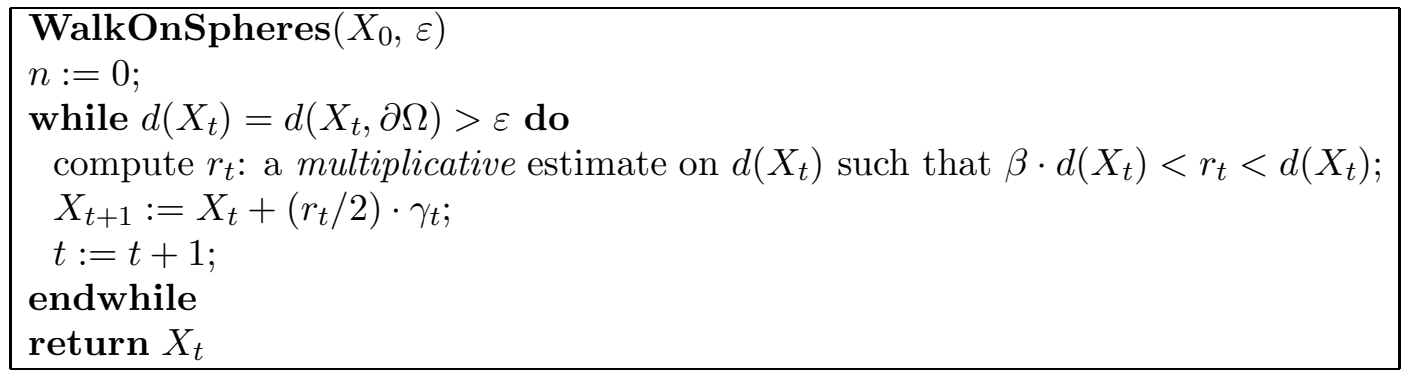

Thus at each step of the algorithm we jump at least $\beta / 2$ and at most $1 / 2$-fraction of the distance to the boundary in a random direction. An example of running the WoS algorithm in 2-d is illustrated on Figure 1.
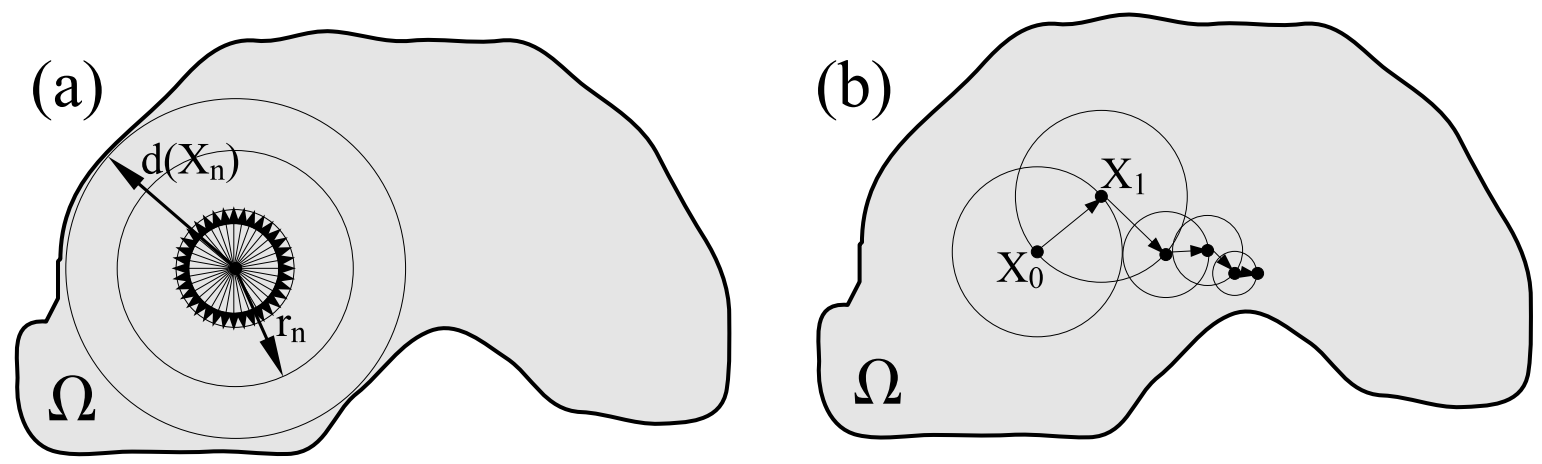

Figure 1. An illustration of the WoS algorithm for $d=2$ : one step jump (a), and a possible run of the algorithm for several steps (b)

As mentioned earlier, it is clear that the algorithm is correct. Moreover, it follows from the usual properties of martingales (see [KS91]) that it converges in $O\left(1 / \varepsilon^{2}\right)$ steps. However, in many 
situations, this rate of convergence is unsatisfactory. In particular, if we wanted to get $2^{-n}$-close to the boundary, it would take us a number of steps exponential in $n$. As it turns out, in many natural situations, the rate of convergence is polynomial or even linear in $n$ (i.e. logarithmic in $1 / \varepsilon$ ). The object of the paper is to prove that this is the case, and give precise condition on when the faster convergence occurs.

While an actual implementation of the WoS would involve round-off errors introduced through an imperfect simulation, we will ignore those to simplify the presentation as they do not affect any of the main results. Thus the problem becomes purely that of analyzing the family of stochastic processes $\left\{X_{t}\right\}$ and their convergence speed to $\partial \Omega$.

Providing the domain $\Omega$ to the algorithm. It is worth noting that the algorithm needs access to the input domain $\Omega$ in a very weak sense. We need an oracle $\operatorname{dist}_{\Omega}(x)$ that satisfies the following:

$$
\operatorname{dist}_{\Omega}(x) \in \begin{cases}(\beta d(x), d(x)) & \text { if } x \in \Omega, d(x)>\beta \varepsilon \\ {[0, \beta \varepsilon)} & \text { if } x \in \Omega, d(x) \leq \beta \varepsilon \\ 0 & \text { if } x \notin \Omega\end{cases}
$$

for some $0<\beta<1$. Note that $\operatorname{dist}_{\Omega}$ would also allow us to decide both the size of the jump on step $t$ and whether $X_{t}$ is sufficiently close to $\partial \Omega$ for the algorithm to terminate.

If $\Omega$ is given to the algorithm as a union of squares on a $\varepsilon$-fine grid, then dist $\Omega$ can be computed in time poly $(1 / \varepsilon)$. In many applications, however, this function can be computed in time poly $(\log 1 / \varepsilon)$, because we only need to estimate the distance within a multiplicative error of $\beta$. The precise condition for this is that the complement set $\Omega^{c}$ is polynomial-time computable as a subset of $\mathbb{R}^{d}$ in the sense of Computable Analysis. See for example [BW99, Wei00, BC06] for more details on polynomial-time computability of real sets. The vast majority of domains in applications satisfy this condition.

Thus, in cases when the domain $\Omega$ is sufficiently nice for $\Omega^{c}$ to be polynomial-time computable, the rate of convergence of the WoS becomes the crucial component in the running time of its execution. In particular, depending on whether the rate of convergence is poly $(1 / \varepsilon)$ of poly $(\log 1 / \varepsilon)$ it could take time that is either exponential or polynomial in $n$ to sample points that are $2^{-n}$-away from $\partial \Omega$.

1.2. Prior work. The WoS algorithm was first proposed in 1956 by M. Muller in [Mul56]. As we have mentioned earlier, it is not hard to see that it takes at most $O\left(1 / \varepsilon^{2}\right)$ steps for the WoS process to reach an $\varepsilon$-neighborhood of $\partial \Omega$. It is also obvious that the algorithm takes $\Omega(\log 1 / \varepsilon)$ steps to finish, since the distance to the boundary is at most halved at each step. It turns out that in many situations this simple lower bound is tight.

Logarithmic rate of convergence of the process $X_{t}$ to the boundary was established for convex domains by M. Motoo in [Mot59]. It was later generalized by G.A. Mikhailov in [Mih79] to planar domains satisfying any cone condition (i.e. at every point of the boundary there is a cone of certain fixed opening in the complement of the domain), as well for 3-dimensional domains satisfying a cone condition with large enough surface angle. See also [EKMS80] and [Mil95] for additional historical background and the use of the algorithm for solving various types of boundary value problems.

In our earlier work [BB07], we established polylogarithmic, but not logarithmic, upper bounds on the rate of convergence of WoS for planar domains, and for a restricted class of higher-dimensional domains. Unfortunately, the techniques of [BB07] do not generalize well to general domains in higher dimensions.

Our present results subsume all prior work on the rate of convergence of the WoS. We introduce an easily verified dimensional condition on the domain which provides tight bounds for the rates of convergence. We also show that the condition is tight. For example, we exhibit planar domains for which the rate of convergence is $\Omega\left(\log ^{2} 1 / \varepsilon\right)$. 
1.3. Results. Let $H^{\beta}(K)$ denote the $\beta$-dimensional Hausdorff content of $K$, which is given by the following formula:

$$
H^{\beta}(K)=\inf _{K \subset \cup B\left(x_{j}, r_{j}\right)} \sum r_{j}^{\beta} .
$$

Intuitively, $H^{\beta}(K)$ measures the volume of the set $K$, when it is viewed as a $\beta$-dimensional set. For example, if $K$ is the two dimensional unit disc, then $H^{3}(K)=0$, but $H^{2}(K)>0$.

We use Hausdorff content to introduce the notion of $\alpha$-thickness.

Definition 1. A domain $\Omega \subset \mathbb{R}^{d}$ is said to be $\alpha$-thick $0 \leq \alpha \leq d$ if there exists a constant $C>0$ such that for every $x \in \partial \Omega$

$$
H^{d-\alpha}(B(x, r) \backslash \Omega) \geq C r^{d-\alpha}, \quad r<1 .
$$

Roughly speaking, $\alpha$-thick domains have complements of codimension $\alpha$, which are uniformly large at every scale at every boundary point.

We call the constant $C$ the thickness of the domain $\Omega$. It is not hard to see that the property of $\alpha$-thickness is monotone: an $\alpha$-thick domain is $\alpha^{\prime}$-thick for $\alpha<\alpha^{\prime} \leq d$.

Let us list some examples of $\alpha$-thick domains.

(1) All $d$-dimensional domains are $d$-thick;

(2) all bounded $d$-dimensional domains $\Omega$ such that the complement $\Omega^{c}$ is connected are $d-1$ thick.

(3) all convex domains and all domains satisfying any cone condition are 0-thick;

(4) all domains $\Omega$ that are bounded by a smooth hypersurface $\partial \Omega$ are 0 -thick.

For example, if $\Omega$ is a domain such that the complement $\Omega^{c}$ is connected, then each point $x \in \partial \Omega$ is connected to $\infty$ via a 1 -dimensional curve, thus implying $d-1$-thickness.

It turns out that the $\alpha$-thickness of the domain is responsible for the rate of convergence of the WoS algorithm. This idea is formulated precisely in our Main Theorem.

Theorem 2. Let $\Omega$ be a bounded $\alpha$-thick domain in $\mathbb{R}^{d}$. Then the expected rate of convergence of the $W o S$ from any $x \in \Omega$ until termination at distance $<\varepsilon$ to the boundary is given by the following table:

\begin{tabular}{|c|c|}
\hline & Rate of convergence \\
\hline \hline$\alpha<2$ & $O(\log 1 / \varepsilon)$ \\
\hline$\alpha=2$ & $O\left(\log ^{2} 1 / \varepsilon\right)$ \\
\hline$\alpha>2$ & $O\left((1 / \varepsilon)^{2-4 / \alpha}\right)$ \\
\hline
\end{tabular}

The $O(\cdot)$ in the expressions above depends on the dimension $d$, on $\alpha$, on the thickness constant $C$ from Definition 1 and on $\beta>0$ from the definition of the WoS. It does not depend directly on $\Omega$ or on the choice of initial point $X_{0}$.

Moreover, the rates of convergence above are tight. Formally, let $f_{\alpha}(1 / \varepsilon)$ be the growth rate asymptotically given by the formulas in (3). For any $\alpha$ and for any $g(1 / \varepsilon)$ such that $g(1 / \varepsilon)=$ $o\left(f_{\alpha}(1 / \varepsilon)\right)$, there is an $\alpha$-thick domain $\Omega_{g}^{\alpha}$ with some thickness $C$ such that the rate of convergence of the WoS algorithm on $\Omega_{g}^{\alpha}$ is asymptotically slower than $g(1 / \varepsilon)$ for a sequence $\left\{\varepsilon_{n}\right\}_{n=1}^{\infty}$ of $\varepsilon$ 's that converge to 0 .

Remark 3. Note that there are several equivalent ways of defining the rate of convergence of the WoS algorithm. For example, the rate of convergence is $\lambda$ if for each starting point $z$,

$$
\mathbf{P}[\text { the first } \varepsilon \text {-convergence time is before } \lambda]>1 / 2 \text {. }
$$

Since our results do not depend on the starting point, the following definition will be equivalent up to a constant:

$$
\mathbf{E}[\text { the first } \varepsilon \text {-convergence time }]<2 \lambda \text {. }
$$


Moreover, the convergence time decays exponentially:

$$
\mathbf{P}[\text { the first } \varepsilon \text {-convergence time is before } k \cdot \lambda]>1-1 / 2^{k} \text {. }
$$

Recall that the rate of convergence cannot be better than $\Omega(\log 1 / \varepsilon)$. An intuitive explanation to the phase transition phenomenon occurring at $\alpha=2$, is that a BM in $\mathbb{R}^{d}$ almost surely "misses" sets of co-dimension $>2$, while hitting sets of co-dimension $<2$ with positive probability.

It is worth noting that the main result in [BB07] is the special case $\alpha=d=2$ of the theorem.

The following corollaries are implied directly by the Theorem 2, and subsume all prior results on the convergence rate of the WoS.

Corollary 4. (1) Since any planar domain is 2-thick, the WoS converges in $O\left(\log ^{2} 1 / \varepsilon\right)$ steps;

(2) since any planar domain with connected exterior is 1 -thick, the WoS converges in $O(\log 1 / \varepsilon)$ steps;

(3) since any domain in $\mathbb{R}^{d}$ is $d$-thick, for $d \geq 3$ the WoS converges in $O\left((1 / \varepsilon)^{2-4 / d}\right)$ steps;

(4) since any 3-dimensional domain with connected exterior is 2-thick, the WoS converges in $O\left(\log ^{2} 1 / \varepsilon\right)$ steps;

(5) since for any $d \geq 4$, any $d$-dimensional domain with connected exterior is $d$ - 1-thick, the WoS converges in $O\left((1 / \varepsilon)^{2-4 /(d-1)}\right)$ steps;

(6) since any domain bounded by a smooth hypersurface is 0-thick, the WoS converges in $O(\log 1 / \varepsilon)$ steps.

The rest of the paper is organized as follows. In Section 2 we construct the auxiliary boundary barrier measures and the energy functions. Using these functions, we prove the upper estimates of Theorem 2. More technical estimates on the energy function are done in Section 3. Finally, in Section 4, we present examples of $\alpha$-thick domains with the slow rate of convergence of the WoS process.

Acknowledgments. We would like to thank Stephen Cook and Greg Lawler for their valuable suggestions. We would also like to thank anonymous referee for a multitude of helpful comments which served to correct the exposition.

\section{UPPER BOUNDS: ENERGY FUNCTIONS}

2.1. Construction of an auxiliary measure. In this section we will construct a family of (finite but not necessarily probability) measures near boundary points of an $\alpha$-thick domain. These measures will be used to construct energy functions, which, in turn, play a crucial role in the proof of Theorem 2 .

Lemma 5. There exists a constant $c=c(\alpha, d, C)$ such that for any $\alpha$-thick domain $\Omega$ with thickness $C$ in $\mathbb{R}^{d}$ and for any $x \in \partial \Omega$, one can find a Borel measure $\mu_{x}$ which satisfies the following conditions:

(1) $\operatorname{supp}\left(\mu_{x}\right) \cap \Omega=\emptyset$, or, equivalently, $\mu_{x}(\Omega)=0$;

(2) for any $y \in \mathbb{R}^{d}$ and $r>0, \mu_{x}(B(y, r)) \leq r^{d-\alpha}$;

(3) for any $r<1, \mu_{x}(B(x, r)) \geq c \cdot r^{d-\alpha}$.

With a slight abuse of notation, we will also refer to the constant $c$ from this lemma as the thickness of the domain.

Proof. The proof of the Lemma follows the standard reasoning that can be found in, say, Chapter II of [Car67].

Let us consider the dyadic grid selected so that the point $x$ has coordinates $(1 / 3,1 / 3, \cdots, 1 / 3)$. For an integer $d$-multi-index $\gamma=\left(\gamma_{1}, \ldots, \gamma_{d}\right)$, let $D_{k, \gamma}$ be the cube

$$
\left\{\left(x_{1}, \ldots, x_{d}: \gamma_{n} 2^{-k} \leq x_{n}<\left(\gamma_{n}+1\right) 2^{-k}, n=1, \ldots, d\right\} .\right.
$$


Let $D_{k}(x)$ be the unique dyadic cube of the size $2^{-k}$ which contains $x$. Note that $x$ is always at distance $2^{-k} / 3$ from the boundary of $D_{k}(x)$.

We will construct inductively a sequence of measures $\nu_{n}$. They will satisfy the following properties:

(a) $\operatorname{supp} \nu_{n} \cap \Omega=\emptyset$.

(b) $\nu_{n}\left(D_{k, \gamma}\right) \leq H^{d-\alpha}\left(D_{k, \gamma} \backslash \Omega\right)$ for $1 \leq k \leq n$ and for all multi-indexes $\gamma$.

(c) $\nu_{n}\left(D_{k}(x)\right)=H^{d-\alpha}\left(D_{k}(x) \backslash \Omega\right)$ for $1 \leq k \leq n$

Let $\nu_{1}$ be a delta measure in a point of $D_{1}(x) \backslash \Omega$ with the total mass $H^{d-\alpha}\left(D_{1}(x)\right)$. It clearly satisfies all of our assumptions.

Assume now that the measure $\nu_{n}$ has already been constructed. The measure $\nu_{n+1}$ will be a sum of delta-measures on the points outside of $\Omega$ lying in the cubes from the $n+1$-st dyadic generation, such that $\nu_{n+1}\left(D_{n, \gamma}\right)=\nu_{n}\left(D_{n, \gamma}\right)$ for all $\gamma$ (so $\nu_{n+1}$ will be obtained from $\nu_{n}$ by re-distributing the latter over the cubes of the $(n+1)$-st generation). Thus the measure $\nu_{n+1}$ would automatically satisfy the second and the third condition for $k \leq n$.

To construct $\nu_{n+1}$, we use the following rule.

First, we set $\nu_{n+1}\left(D_{n+1}(x)\right)=H^{d-\alpha}\left(D_{n+1}(x) \backslash \Omega\right)$. The measure $\nu_{n+1}$ clearly satisfies our condition (c) on $D_{n+1}(x)$.

Second, for any other dyadic cubes $D_{n+1, \gamma} \subset D_{n}(x)$, we assign the mass

$$
\nu_{n+1}\left(D_{n+1, \gamma}\right)=H^{d-\alpha}\left(D_{n+1, \gamma} \backslash \Omega\right) \frac{\left(\nu_{n}\left(D_{n}(x)\right)-\nu_{n+1}\left(D_{n+1}(x)\right)\right)}{\sum_{D_{n+1, \delta} \subset D_{n}(x), D_{n+1, \delta} \neq D_{n+1}(x)} H^{d-\alpha}\left(D_{n+1, \delta} \backslash \Omega\right)},
$$

so that $\nu_{n}\left(D_{n}(x)\right)=\nu_{n+1}\left(D_{n}(x)\right)$. By sub-additivity of the Hausdorff content,

$$
\left.\sum_{D_{n+1, \delta} \subset D_{n}(x)} H^{d-\alpha}\left(D_{n+1, \delta} \backslash \Omega\right)\right) \geq H^{d-\alpha}\left(D_{n}(x)\right)
$$

and hence

$$
\nu_{n+1}\left(D_{n+1, \gamma}\right) \leq H^{d-\alpha}\left(D_{n+1, \gamma} \backslash \Omega\right)
$$

for $D_{n+1, \gamma} \subset D_{n}(x)$.

Finally, for any other dyadic cubes from $(n+1)$-st generation, we set

$$
\nu_{n+1}\left(D_{n+1, \gamma}\right)=H^{d-\alpha}\left(D_{n+1, \gamma} \backslash \Omega\right) \frac{\nu_{n}\left(D_{n}\right)}{\sum_{D_{n+1, \delta} \subset D_{n}} H^{d-\alpha}\left(D_{n+1, \delta} \backslash \Omega\right)},
$$

where $D_{n}$ is the unique cube from the $n$-th dyadic generation containing $D_{n+1, \gamma}$. Using the subadditivity of the Hausdorff content, as above, we get the estimate (5) for all cubes of the $(n+1)$-st generation. The construction again satisfies $\nu_{n+1}\left(D_{n}\right)=\nu_{n}\left(D_{n}\right)$.

Let now $\nu$ be any weak* limit point of the sequence $\nu_{n} . \nu$ is still supported outside of $\Omega$. By the second property of the measures $\nu_{n}$,

$$
\nu\left(D_{k, \gamma}\right) \leq \sum_{\overline{D_{k, \delta}} \cap \overline{D_{k, \gamma}} \neq \emptyset} H^{d-\alpha}\left(D_{k, \delta} \backslash \Omega\right) \leq 3^{d} H^{d-\alpha}\left(D_{k, \gamma}\right)<3^{d}(\sqrt{d})^{d} 2^{-k(d-\alpha)}
$$

for all $k$. Using the third property of the measures $\nu_{n}$, the $\alpha$-thickness of $\Omega$, and the fact that $D_{k}(x)$ contains the ball of the radius $2^{-k} / 3$, we get that

$$
\nu\left(D_{k}(x)\right) \geq H^{d-\alpha}\left(D_{k}(x) \backslash \Omega\right) \geq c 3^{-d} 2^{-k(d-\alpha)}
$$

for any $k$.

Every ball can be covered by certain ( $d$-dependent) number of dyadic cubes of comparable size, so (7) implies that $\nu(B(y, r))<K r^{d-\alpha}$ for some $K>0$. Every ball centered at $x$ also contains a 
dyadic cube of comparable (again, $d$-dependent) size, hence by $(8), \nu(B(x, r))>k r^{d-\alpha}$ for some $k>0$. Now we can set $\mu_{x}$ to be an appropriately normalized measure $\nu$.

2.2. Energy Function of optimal growth. The heart of the proof of the upper bounds in Theorem 2 is the construction of a subharmonic function with optimal (for our problem) growth at the boundary, the Energy Function $U$ on $\Omega$. We will construct $U(x)$ so that it is "small" in the interior of $\Omega$, and grows to $\infty$ as $x$ approaches the boundary $\partial \Omega$. The $\alpha$-thickness of the domain allows us to establish that the value of $U\left(X_{t}\right)$ grows in expectation as the WoS progresses. Thus after a certain number of steps $U\left(X_{t}\right)$ will be large in expectation which would imply that $X_{t}$ is close to $\partial \Omega$ with high probability. Let us note that the construction of the Energy Function is pretty similar to the classical construction used, for example, in the proof of the Wiener regularity criterion (see [Lan72]).

The construction of the function is based on the notion of a Riesz potential. For a finite Borel measure $\mu$ on $\mathbb{R}^{d}$, and $\alpha<d$, the $\alpha$-Riesz potential of the measure $\mu$ is defined by

$$
U_{\alpha}^{\mu}(x)=\frac{1}{d-\alpha} \int \frac{d \mu(z)}{|z-x|^{d-\alpha}} .
$$

For $\alpha=d$, the $d$-Riesz potential is defined by

$$
U_{\alpha}^{\mu}(x)=\int \log \frac{1}{|z-x|} d \mu(z) .
$$

The value $U_{\alpha}^{\mu}(x)=\infty$ is allowed when the integral diverges.

An important special case is the case of $\alpha=2$, the so-called Newton potential. We will denote $U_{2}^{\mu}$ simply by $U^{\mu}$. In this case, the expression under the integral is harmonic in $\mathbb{R}^{d}$. It is well known (e.g. see [Lan72]) that the function $U^{\mu}$ is superharmonic on $\mathbb{R}^{d}$, and harmonic outside of $\operatorname{supp} \mu$.

More generally, outside of the supp $\mu$, we have the identity

$$
\Delta U_{\alpha}^{\mu}(y)=(d-\alpha+2)(2-\alpha) U_{\alpha-2}^{\mu}(y) .
$$

It shows that for $0<\alpha<2$, the function $U_{\alpha}^{\mu}$ is subharmonic outside of $\operatorname{supp} \mu$.

The following important technical identity, which easily follows from Fubini's Theorem and substitution, relates the local behavior of the measure $\mu$ and the growth of its potential $U_{\alpha}^{\mu}$. For $\alpha<d$, we have

$$
U_{\alpha}^{\mu}(y)=\int_{0}^{\infty} \frac{\mu(B(y, r))}{r^{d-\alpha+1}} d r
$$

and for $\alpha=d$,

$$
U_{\alpha}^{\mu}(y)=\lim _{R \rightarrow \infty}\left(\int_{0}^{R} \frac{\mu(B(y, r))}{r} d r-\mu(B(y, R)) \log R\right)
$$

Let us now fix an $\alpha$-thick domain $\Omega \subset B(0,1) \subset \mathbb{R}^{d}$. Let us consider the set $\mathcal{M}$ of all Borel measures $\mu$ supported inside $\overline{B(0,2)}$ and outside of $\Omega$ (i.e. $\mu(\Omega)=0$ ), satisfying the following condition:

$$
\text { for any } y \in \mathbb{R}^{d} \text { and } r>0, \mu(B(y, r)) \leq r^{d-\alpha}
$$

Let us now introduce the Energy Function $U(y)$. Recall that we denote $U^{\mu}(y):=U_{2}^{\mu}(y)$.

$$
U(y):= \begin{cases}\sup _{\mu \in \mathcal{M}} U_{\alpha}^{\mu}(y), & \text { when } \alpha \leq 2 \\ \sup _{\mu \in \mathcal{M}} U^{\mu}(y), & \text { when } \alpha \geq 2 .\end{cases}
$$

Since the set $\mathcal{M}$ is weakly*-compact, for every $y \in \Omega$ there exists a measure maximizing the potential in (13) at the point $y$. 
Let us summarize the properties of $U(y)$ in the following claim. The proof uses the identities (10) and (11). Recall that $d(y)=\operatorname{dist}(y, \partial \Omega)$.

Claim 6. Let $\Omega$ be an $\alpha$-thick domain. Then

(1) $U(y)$ is subharmonic in $\Omega$.

(2) For $\alpha \leq 2, U(y) \leq \log \frac{3}{d(y)}+K(d, \alpha)$ for all $y \in \Omega$. Here $K(2,2)=0$, and $K(d, \alpha)=\frac{1}{d-\alpha}$ when $d \neq \alpha$.

(3) For $\alpha>2, U(y) \leq \frac{1}{\alpha-2} d(y)^{2-\alpha}$ for all $y \in \Omega$.

Proof. Let $\alpha \leq 2, y \in \Omega$ and $\mu \in \mathcal{M}$. Equations (10) and imply that when $d>\alpha$

$$
U_{\alpha}^{\mu}(y) \leq \int_{d(y)}^{3} \frac{1}{t} d t+\int_{3}^{\infty} \frac{\mu\left(\mathbb{R}^{d}\right)}{t^{d-\alpha+1}} d t \leq \log \frac{3}{d(y)}+\frac{1}{d-\alpha} .
$$

When $d=\alpha=2$, by (11) the last constant $\frac{1}{d-\alpha}$ disappears. Similarly, for $\alpha>2$, we will use the harmonic potential $U^{\mu}$. Let $\alpha>2, y \in \Omega$ and $\mu \in \mathcal{M}$. Once again, (12), supp $\mu \cap \Omega=\emptyset$, and the equations (10) imply that

$$
U^{\mu}(y) \leq \int_{d(y)}^{\infty} \frac{1}{t^{\alpha-1}} d t \leq \frac{1}{(\alpha-2) d(y)^{\alpha-2}} .
$$

Equation (14) and (15) imply that the potentials $U^{\mu}$ are locally uniformly bounded. Thus, for $\alpha \geq 2, U(y)$ is a continuous subharmonic function as a supremum of a locally bounded family of harmonic functions.

For $\alpha<2$, let us observe that

$$
\begin{aligned}
\left|\nabla U_{\alpha}^{\mu}(y)\right| \leq \frac{1}{d-\alpha} \int\left|\nabla \frac{1}{|\xi-y|^{d-\alpha}}\right| d \mu(\xi)=\int \frac{1}{|\xi-y|^{d-\alpha+1}} d \mu(\xi)= \\
\quad(d-\alpha+1) \int_{d(y)}^{\infty} \frac{\mu(B(y, r))}{r^{d-\alpha+2}} d r \leq(d-\alpha+1) \int_{d(y)}^{\infty} \frac{r^{d-\alpha}}{r^{d}-\alpha+2} d r \leq \frac{A}{d(y)}
\end{aligned}
$$

for some constant $A$ depending on $d$ and $\alpha$. We use (14) and the preceding estimate to see that the family $\left\{U_{\alpha}^{\mu}: \mu \in \mathcal{M}\right\}$ is locally equicontinuous. Thus, since each function $U_{\alpha}^{\mu}$ is subharmonic, the function $U(y)$ is also continuous and subharmonic.

The second and third statements of the claim follow directly from (14) and (15) respectively.

Let $X_{t}$ be the WoS process initiated at some point $X_{0}=y \in \Omega$. Let us define a new process $U_{t}=U\left(X_{t}\right)$, the value of the energy function at the $t$-th step of the process. Note that because $U$ is subharmonic, $U_{t}$ is a submartingale, that is $\mathbf{E}\left[U_{t+1} \mid U_{t}\right] \geq U_{t}$.

Claim 6 immediately implies that a large value of $U_{t}$ will guarantee closeness to the boundary. More specifically,

Claim 7. For $\alpha \leq 2$, if $U_{t}>\log \frac{2}{\varepsilon}$ then $d\left(X_{t}\right)<\varepsilon$.

For $\alpha>2, U_{t}>(\alpha-2) \varepsilon^{2-\alpha}$ implies $d\left(X_{t}\right)<\varepsilon$.

The proof of Theorem 2 relies on finer lower bounds on the function $U$, which would guarantee the optimal rate of boundary convergence. We prove the bounds in the next section. These bounds depend heavily on the value of $\alpha$. We first give a probabilistic proof of the upper bounds in Theorem 2 , and then prove the finer estimates on $U$ in Section 3.

For $\varepsilon>0$, we denote by $T_{\varepsilon}:=\min \left\{t: d\left(X_{t}\right)<\varepsilon\right\}$, the first time the process $X_{t}$ hits the $\varepsilon$-neighborhood of the boundary. 
2.3. Logarithmic convergence: the case $\alpha<2$. In the heart of the proof for this case lies the following strong estimate on the behavior of the Riesz potentials near the boundary.

Lemma 8. For any $\alpha<2$ and $c>0$, there exist two constants $\delta$ and $\eta$, such that the following holds.

Let $\Omega$ be an $\alpha$-thick domain in $\mathbb{R}^{d}$ with thickness $c$. Let $y \in \Omega$ and $x \in \partial \Omega$ be a closest point to y. Let $\mu \in \mathcal{M}$.

Then either

$$
U(z)>U_{\alpha}^{\mu}(z)+1 \text { whenever }|z-x|<\delta \cdot d(y) \text { and } d(z)>\delta / 4 \cdot d(y) .
$$

or

$$
\mu(B(y, 2 d(y))) \geq \eta d(y)^{d-\alpha}
$$

The lemma is established in Section 3.1.

Note that after $k=O(|\log \delta|)$ steps of the WoS process,

$$
\left|X_{t+k}-x\right|<\delta \cdot d\left(X_{t}\right) \text { and } \delta / 4 \cdot d\left(X_{t}\right)<d\left(X_{t+k}\right) \text { with a certain probability } p,
$$

where $x$ is the point of $\partial \Omega$ that is closest to $X_{t}$, and $p>0$ depends only on $\beta$ and the dimension $d$.

Let us fix $X_{t}$ and take the measure $\mu \in \mathcal{M}$ maximizing the value of $U_{\alpha}^{\mu}\left(X_{t}\right)$. By the preceding observation, in the first case in Lemma 8, the subharmonicity of $U_{\alpha}^{\mu}$ implies that the expectation of $U_{t+k}$, conditioned on $U_{t}$, will increase by some definite constant.

On the other hand, using the identity (9) and the $\alpha$-thickness of $\Omega$, one can see that the Laplacian of $U_{\alpha}^{\mu}$ is large near the point $X_{t}$ in the second case of Lemma 8. Thus, since large Laplacian leads to a fast build-up of mean values, we have the above-mentioned increase by a constant after the first step. We arrive at the following estimate, which shows that $U_{t}$ grows at least linearly in expectation.

Lemma 9. There are constants $L$ and $k$, depending only on $c, \beta$, and $\alpha$, such that

$$
\mathbf{E}\left[\left(U_{t+k}-U_{t}\right) \mid U_{t}\right]>L .
$$

A detailed proof of the lemma can be found in Section 3.2.

Lemma 9 implies that $\mathbf{E}\left[U_{t}\right]>t L / k+U_{0}$. Since $d\left(X_{t}\right) \geq(1-\beta)^{t} d\left(X_{0}\right)$, Claim 6 implies that $U_{t} \leq \log \frac{2}{d\left(X_{0}\right)}+t|\log (1-\beta)|+\log 2$. This implies that $U_{t}>\log \frac{2}{d\left(X_{0}\right)}+t L / 2 k$ with probability at least $P$, where $P$ depends only on $\beta$. This, together with Claim 7 implies the necessary upper bound in the case $\alpha<2$.

2.4. Polylogarithmic convergence: the case $\alpha=2$. In the case $\alpha=2$ the steady linear growth of $U_{t}$ given by Lemma 9 no longer holds. In fact, the only thing that generally holds in this case is the submartingale property $\mathbf{E}\left[U_{t+1} \mid U_{t}\right] \geq U_{t}$. We are able to overcome this difficulty by showing that the submartingale process $\left\{U_{t}\right\}$ has a deviation bounded from below by a constant at every step. To this end it suffices to show that $U_{t}$ can grow by some $\eta$ with a non-negligible probability. We use the following estimate on the energy function (established in Section 3.1).

Lemma 10. There exists a constant $\delta$, dependent only on the thickness $c$, such that the following holds. Let $\Omega$ be a 2-thick domain. Let $y \in \Omega$ and $x \in \partial \Omega$ be the closest point to $y$. Then

$$
U(z)>U(y)+1 \text { whenever }|z-x|<\delta \cdot d(y) .
$$

Since the function $U$ is subharmonic, observation (18) implies the following estimate (see Section 3.2 for a proof).

Lemma 11. Let $\Omega$ be a 2-thick domain in $\mathbb{R}^{d}$. There are constants $k$ and $L$, depending only on the thickness $c$, the jump ratio $\beta$, and the dimension $d$, such that

$$
\mathbf{E}\left[\left(U_{t+k}-U_{t}\right)^{2} \mid U_{t}\right]>L .
$$


We can now use Lemma 11 to prove the upper bounds on the rate of convergence for the case $\alpha=2$. Fix $\varepsilon>0$ and denote by $\varepsilon^{\prime}:=2^{-k} \varepsilon$. We replace the submartingale $U_{t}$ by a stopped submartingale

$$
V_{t}=\left\{\begin{array}{ll}
U_{t}, & t<T_{\varepsilon^{\prime}} \\
U_{T_{\varepsilon^{\prime}}}, & t \geq T_{\varepsilon^{\prime}}
\end{array},\right.
$$

By the optional stopping time theorem (see [KS91]), $V_{t}$ is also a positive submartingale. In addition, it is a bounded submartingale: $V_{t} \leq \log \frac{2^{k+2}}{\varepsilon}$. Since $V_{t}$ is a submartingale, we also have

$$
\mathbf{E}\left[V_{t}\left(V_{t+k}-V_{t}\right)\right]=\mathbf{E}\left[\mathbf{E}\left[V_{t}\left(V_{t+k}-V_{t}\right) \mid V_{t}\right]\right] \geq \mathbf{E}\left[\mathbf{E}\left[V_{t}\left(V_{t}-V_{t}\right) \mid V_{t}\right]\right]=0
$$

If $t<T_{\varepsilon}$ then, by the choice of $\varepsilon^{\prime}, U_{t}=V_{t}$ and $U_{t+k}=V_{t+k}$. Thus Lemma 11 implies that

$$
\mathbf{E}\left[\left(U_{t+k}-U_{t}\right)^{2}\right] \geq \mathbf{E}\left[\mathbf{E}\left[U_{t+k}-U_{t} \mid U_{t}\right] \cdot \mathbb{1}_{T_{\varepsilon}>t}\right]>L \cdot \mathbf{P}\left[T_{\varepsilon}>t\right] .
$$

We are now in a position to establish the upper bounds for $\alpha=2$.

Proof of the upper bound from Theorem 2 for $\alpha=2$. Assume first that for some M,

$$
\mathbf{P}\left[T_{\varepsilon}>M \log ^{2} \frac{1}{\varepsilon}\right] \geq 1 / 2 \text {. }
$$

It means that for all $t \leq M \log ^{2} \frac{1}{\varepsilon}, \mathbf{P}\left[T_{\varepsilon} \geq t\right] \geq 1 / 2$. This implies

$$
\left.\mathbf{E}\left[V_{t+k}^{2}\right]=\mathbf{E}\left[\left(V_{t+k}-V_{t}\right)+V_{t}\right)^{2}\right]=\mathbf{E}\left[V_{t}^{2}\right]+\mathbf{E}\left[\left(V_{t+k}-V_{t}\right)^{2}\right]+2 \mathbf{E}\left[V_{t}\left(V_{t+k}-V_{t}\right)\right] \geq \mathbf{E}\left[V_{t}^{2}\right]+L / 2 .
$$

The last inequality follows from (20) and (21). By the previous inequality,

$$
\mathbf{E}\left[V_{M \log ^{2} \frac{1}{\varepsilon}}^{2}\right] \geq \frac{L M \log ^{2} \frac{1}{\varepsilon}}{k}
$$

Since $V_{t} \leq \log \frac{2^{k+2}}{\varepsilon}$, this leads to a contradiction for large enough $M$.

2.5. Polynomial convergence: the case $\alpha>2$. For the case $\alpha>2$, the required converse to Claim 6 is relatively simple.

Lemma 12. For $\alpha>2$, and an $\alpha$-thick domain $\Omega$ in $\mathbb{R}^{d}$ with the thickness $c$,

$$
U(y) \geq K \cdot c \cdot d(y)^{2-\alpha}
$$

for all $y \in \Omega$. Here the constant $K=K(\alpha)$ depends only on $\alpha$.

The lemma is established in Section 3.1.

The idea of the proof of Theorem 2 in this case is now as follows. When the WoS is far from the boundary $\partial \Omega$ it makes fairly big steps and when it is close it makes small steps. There are not too many big steps because the number of big steps of length $>\varepsilon$ confined to $B(0,1)$ is bounded by $O\left(1 / \varepsilon^{2}\right)$ (in expectation). On the other hand, there are not too many small steps, because a small step means that the WoS is very close to $\partial \Omega$, and should converge before an opportunity to make many more steps.

More precisely, the number of "big" jumps is bounded by the following claim.

Claim 13. Let $N(\delta, T)$ be the number of the jumps in the WoS process before the time $T$ which are bigger than $\delta$, i.e.

$$
N(\delta, T)=\#\left\{t \leq T|| X_{t}-X_{t-1} \mid \geq \delta\right\} .
$$

Then

$$
\mathbf{P}\left[N(\delta, T)>\frac{4}{\delta^{2}}\right]<1 / 4
$$


Proof. Note now that because $X_{t}$ is a martingale, we have

$$
\begin{aligned}
1 \geq \mathbf{E}\left[X_{T}^{2}\right]-X_{0}^{2}=\sum_{k=1}^{T}\left(\mathbf{E}\left[X_{t}^{2}\right]-\mathbf{E}\left[X_{t-1}^{2}\right]\right)=\sum_{t=1}^{T} \mathbf{E}\left[\left(X_{t}-X_{t-1}\right)^{2}\right]= \\
\quad \mathbf{E}\left[\sum_{t=1}^{T}\left(X_{t}-X_{t-1}\right)^{2}\right] \geq \delta^{2} \cdot \mathbf{E}[N(\delta, T)]
\end{aligned}
$$

The last equation implies the statement of the claim, by Markov's inequality.

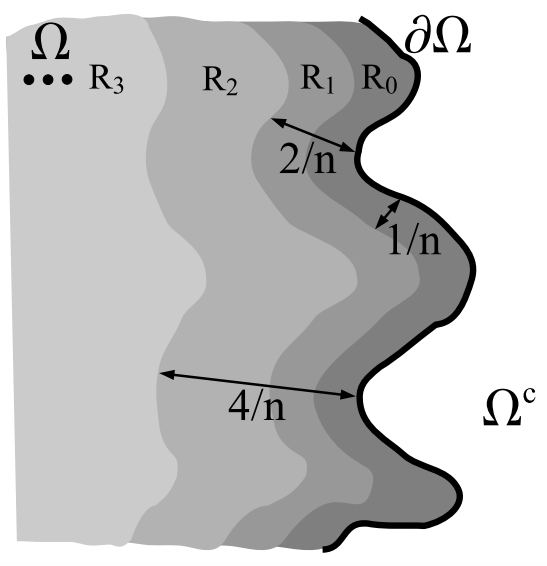

Figure 2. The regions $R_{k}$ from the proof of the upper bounds for $\alpha>2$ with $\varepsilon=1 / n$

To bound the number of small jumps, we denote by $R_{0} \subset \Omega$ the $\varepsilon$-neighborhood of $\partial \Omega$, and more generally, by

$$
R_{k}:=\left\{x \in \Omega: 2^{k-1} \varepsilon<d(x, \partial \Omega) \leq 2^{k} \varepsilon\right\}
$$

(see Figure 2). Note that by Lemma 12 and Claim 6(3), we have

$$
\frac{2^{(k-1)(2-\alpha)}}{\alpha-2} \varepsilon^{2-\alpha} \geq U(y) \geq K 2^{k(2-\alpha)} \varepsilon^{2-\alpha}
$$

for $y \in R_{k}$ and for some $K$, which we take to be much smaller than 1 . Using this fact we prove the following.

Claim 14. Denote by $v_{k}$ the number of visits of $X_{t}$ to $R_{k}$ before the time $T$ when $X_{t}$ first hits the $\varepsilon$-neighborhood of the boundary $\partial \Omega$,

$$
v_{k}=\#\left\{t<T: X_{t} \in R_{k}\right\}
$$

Then

$$
\mathbf{P}\left[v_{k}>C_{1} \cdot 2^{k(\alpha-2)} M\right]<1 / 4^{M},
$$

for some constant $C_{1}=C_{1}(c, d, \alpha, \beta)$ and for any $M>1$.

Proof. Suppose that at some point $t, X_{t} \in R_{k}$. We estimate from below the probability that this is the last time the WoS visits $R_{k}$.

First of all, with some probability $p=p(\alpha, \beta, c, d)>0$ and for some constant $\eta, \mathbf{P}\left[X_{t+\eta} \in\right.$ $\left.R_{k+2} \log (K) /(\alpha-2) \mid X_{t} \in R_{k}\right]>p$, where $K$ is the constant from the estimate eqrefeq:Rk. In other 
words, the series of first jumps brings us much closer to $\partial \Omega$ with definite probability. Consider the subharmonic function

$$
\Phi(y)=(\varepsilon / 2)^{\alpha-2} U(y)-\frac{2^{k(2-\alpha)}}{2-\alpha} .
$$

in $\Omega$. Then the process $\Phi\left(X_{t+j}\right)$ is a submartingale. We stop it at time $t+\tau, \tau \geq \eta$, when either the WoS terminates or when $X_{t+\tau} \in R_{k}$ (i.e. the process gets back to $R_{k}$ ), whichever comes first. If $X_{t+\tau}$ is $\varepsilon$-close to $\partial \Omega$ (but not closer than $\varepsilon / 2$ ), then $\Phi\left(X_{t+\tau}\right) \leq 1$, by (23). If $X_{t+\tau} \in R_{k}$, then, again by (23), $\Phi\left(X_{t+\tau}\right) \leq 0$. Another application of (23) implies that if $y \in R_{k+2 \log (K) /(\alpha-2)}$, then $\Phi(y)>\gamma 2^{k(2-\alpha)}$ for some constant $\gamma>0$. Thus the probability that the WoS terminates at $X_{t+\tau}$ (i.e. we never visit $R_{k}$ again) is at least

$$
\mathbf{P}\left[X_{t+\tau} \notin R_{k}\right] \geq \mathbf{E}\left[\Phi\left(X_{t+\tau}\right)\right] \geq \Phi\left(X_{t+\eta}\right) \geq p \gamma 2^{k(2-\alpha)} .
$$

Thus the probability that the visit $X_{t}$ to $R_{k}$ is the last one is at least $p \cdot \gamma 2^{k(2-\alpha)}$. The claim now follows from an estimate of the probability of having at least $v_{k}$ returns to $R_{k}$, each of them not being the last one.

Claims 13 and 14 together imply the upper bounds on the rate of convergence for $\alpha>2$.

Proof of the upper bounds from Theorem 2 for $\alpha>2$. By Claim 14, for any $k$, we have that

$$
\mathbf{P}\left[v_{k-s}>C_{1} \cdot 2^{(k-s)(\alpha-2)} \cdot(3 / 2+s / 2)\right]<1 / 4^{3 / 2+s / 2}=(1 / 8) \cdot 2^{-s} .
$$

Hence, by union bound $v_{k-s} \leq C_{1} \cdot 2^{(k-s)(\alpha-2)} \cdot(3 / 2+s / 2)$ for all $s \geq 0$ with probability at least $3 / 4$. Let $k$ be such that $2^{k-1} \leq \varepsilon^{-2 / \alpha}<2^{k}$. Then, with the probability at least $3 / 4$, we have the total number of jumps smaller than $2^{k} \varepsilon$ bounded by

$$
\sum_{s=0}^{k} v_{k-s} \leq \sum_{s=0}^{k} C_{1} \cdot 2^{(k-s)(\alpha-2)} \cdot(3 / 2+s / 2)<2^{2(\alpha-2)} /\left(2^{\alpha-2}-1\right) C_{1} \cdot 2^{k(\alpha-2)} \leq C_{1} C_{2} \cdot \varepsilon^{4 / \alpha-2}
$$

for some constant $C_{2}$ which depends only on $\alpha$. If we take $N=2 C_{1} C_{2} \varepsilon^{4 / \alpha-2}$ steps of the WoS, (24) implies that at least half the steps would be of magnitude at least $2^{k} \varepsilon \geq \varepsilon^{1-2 / \alpha}$, except with probability $<1 / 4$. Applying the estimate from Claim 13 with $\delta=2^{k} \varepsilon$, we see that with the probability at least $3 / 4, N\left(2^{k} \varepsilon, t\right) \leq 4 /\left(\varepsilon^{2} 2^{2 k}\right) \leq 4 \varepsilon^{4 / \alpha-2}$. Hence with probability $\geq 1 / 2$ the WoS terminates after $O\left(\varepsilon^{4 / \alpha-2}\right)$ steps.

\section{BOUNDARY BEHAVIOR OF THE ENERGY FUNCTION}

In this section we prove the analytical estimates on the behavior of the energy function that have been used in Section 2.

3.1. Estimating boundary growth. We start with the easiest case $\alpha>2$.

Lemma 12 (Section 2.5): For $\alpha>2$, and an $\alpha$-thick domain $\Omega$ in $\mathbb{R}^{d}$ with the thickness $c$,

$$
U(y) \geq K \cdot c \cdot d(y)^{2-\alpha}
$$

for all $y \in \Omega$. Here the constant $K=K(\alpha)$ depends only on $\alpha$. 
Proof. Let $x$ be a closest to $y$ point at $\partial \Omega$, and let $\mu=\mu_{x}$ be the corresponding measure constructed in Lemma 5. Then, by the identity (10) and since $B(x, r) \subset B(y, r+d(y))$,

$$
\begin{aligned}
U(y) \geq U_{2}^{\mu}(y) \geq \int_{d(y)}^{2} \frac{\mu(B(y, r))}{r^{d-1}} d r \geq \int_{2 d(y)}^{2} \frac{\mu(B(x, r-d(x)))}{r^{d-1}} d r \geq \\
\qquad \int_{2 d(y)}^{2} \frac{\mu(B(x, r / 2))}{r^{d-1}} \geq c 2^{\alpha-d} \int_{2 d(y)}^{2} t^{1-\alpha} \geq K \cdot c \cdot d(y)^{2-\alpha} .
\end{aligned}
$$

Unfortunately, in the case $\alpha \leq 2$ lower bounds of the type established in the proof of Lemma 12 are insufficient, and we will use finer estimates provided by the following construction.

Let $y$ be a point in $\Omega, x$ be a point of $\partial \Omega$ closest to $y$, and $\mu$ be a measure in the class $\mathcal{M}$. We construct a new measure $\nu \in \mathcal{M}$, which we call the amalgamation of $\mu$ at the point $y$ (and $x$, if there are more than one closest points) in the following way.

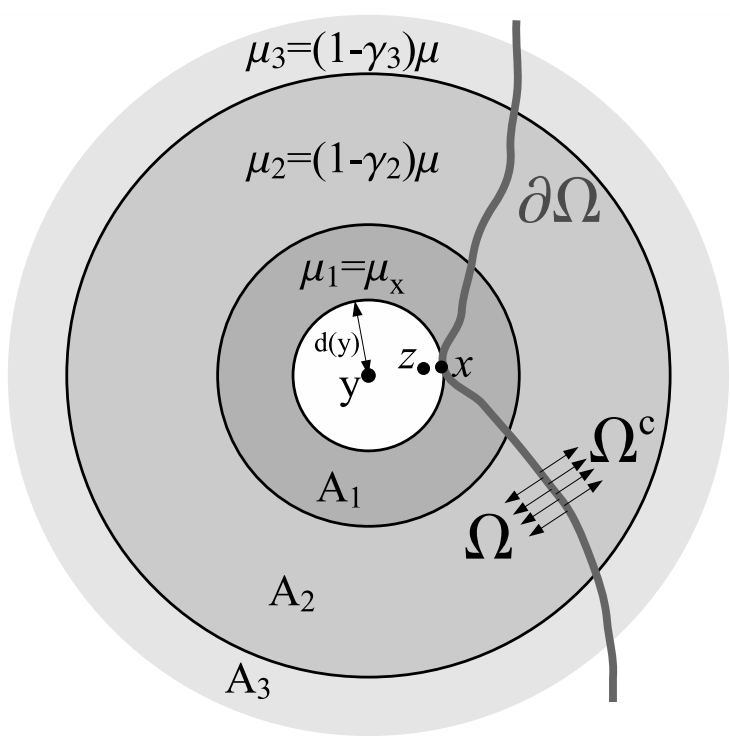

Figure 3. Construction of the amalgamation $\nu=\sum_{k} \mu_{k}$

Let the measure $\mu_{1}$ be the measure $\mu_{x}$ from Lemma 5 restricted to $B(y, 2 d(y)), \mu_{2}=\mu_{3}=0$, and for $k \geq 4$, let $\mu_{k}$ be the measure $\mu$ restricted to the $d$-dimensional annulus

$$
A_{k}=\left\{w: 2^{k-1} d(y) \leq|w-y| \leq 2^{k} d(y)\right\}
$$

scaled by the factor $1-\gamma_{k}:=1-2^{(4-k)(d-\alpha)}$. Let us also put $\gamma_{1}=\gamma_{2}=\gamma_{3}=1$. We define

$$
\nu:=\sum_{k} \mu_{k}
$$

The ingredients of the construction are illustrated on Figure 3.

Let us now prove that $\nu \in \mathcal{M}$. Consider any disk $B(w, r)$. Let $K$ be the largest number such that $B(w, r)$ intersects $A_{K}$. If $B(w, r)$ does not intersect $B(y, 2 d(y))$, the measure $\nu$ is no greater than $\mu$ on $B(w, r)$, and thus $\nu(B(w, r)) \leq r^{d-\alpha}$. If $K \leq 3, \nu(B(w, r)) \leq \mu_{x}(B(w, r)) \leq r^{d-\alpha}$. For 
all other cases, $r \geq 2^{K-3} d(y)$, which, by the choice of $\gamma_{K}$, implies that $\gamma_{K} r^{d-\alpha} \geq(2 d(y))^{d-\alpha}$. Thus

$$
\begin{gathered}
\nu(B(w, r)) \leq \mu_{x}(B(y, 2 d(y)))+\mu(B(w, r))-\sum_{k=1}^{K} \gamma_{k} \mu\left(B(w, r) \cap A_{k}\right) \leq \\
(2 d(y))^{d-\alpha}+\left(1-\gamma_{K}\right) \mu(B(w, r)) \leq \gamma_{K} r^{d-\alpha}+\left(1-\gamma_{K}\right) r^{d-\alpha}=r^{d-\alpha} .
\end{gathered}
$$

The second inequality follows from the fact that the sequence $\left\{\gamma_{k}\right\}$ is non-increasing. We first apply the amalgamation construction to the case $\alpha=2$.

Lemma 10 (Section 2.4): There exists a constant $\delta$, dependent only on the thickness $c$, such that the following holds. Let $\Omega$ be a 2-thick domain. Let $y \in \Omega$ and $x \in \partial \Omega$ be the closest point to $y$. Then

$$
U(z)>U(y)+1 \text { whenever }|z-x|<\delta \cdot d(y) .
$$

Proof. Note that in the case $\alpha=d=2$, we have $U(y)=-\log d(y)$. Thus the statement of the lemma holds with, say, $\delta=1 / 3$.

Let us now consider the case $d>2$. Since $\mathcal{M}$ is a compact set, $U(y)=U^{\mu}(y)$ for some $\mu \in \mathcal{M}$.

Let $\mu_{0}$ be the restriction of the measure $\mu$ to $B(0,2) \backslash B(y, 2 d(y))$. By (10) and the definition of $\mathcal{M}$

$$
U^{\mu_{0}}(y)=U^{\mu}(y)-\int_{d(y)}^{2 d(y)} \frac{\mu(B(y, r))}{r^{d-1}} d r-\int_{2 d(y)}^{\infty} \frac{\mu(B(y, 2 d(y)))}{r^{d-1}} d r \geq U^{\mu}(y)-\log 2-\frac{1}{d-2} .
$$

Let $\nu$ be the amalgamation of $\mu$ at $y$. Next, we will show that

$$
U^{\nu}(z) \geq U^{\mu_{0}}(z)-C_{1}+c \cdot 2^{2-d} \log \frac{1}{\delta}
$$

and

$$
U^{\mu_{0}}(z) \geq U^{\mu_{0}}(y)-C_{2}
$$

whenever $|z-x|<\delta d(y)$ for some constants $C_{1}$ and $C_{2}$ depending only on $d$ and $c$. These inequalities, together with (27), imply the statement of the lemma whenever $\delta$ is sufficiently small (namely, when $\log 1 / \delta>2^{d-2}\left(C_{1}+C_{2}+1+\log 2\right) / c$ ).

To establish (28), let us note that for any $k$ we have

$$
\mu\left(A_{1}\right)+\mu\left(A_{2}\right)+\cdots+\mu\left(A_{k}\right)=\mu\left(B\left(y, 2^{k} d(y)\right)\right) \leq\left(2^{k} d(y)\right)^{d-2} .
$$

By the Abel summation formula,

$$
\begin{aligned}
& \sum_{k \geq 2} \gamma_{k} 2^{k(2-d)} \mu\left(A_{k}\right)=\sum_{k \geq 3} \gamma_{k-1} 2^{(k-1)(2-d)}\left(\mu\left(B\left(y, 2^{k+1} d(y)\right)\right)-\mu\left(B\left(y, 2^{k} d(y)\right)\right)\right) \leq \\
& \sum_{k \geq 3} d(y)^{d-2}\left(2^{d-2} \gamma_{k-1}-\gamma_{k}\right) \cdot 2^{2-d} \leq 5(d(y))^{d-2} .
\end{aligned}
$$

Note that for $w \in A_{k}$ we have $|z-w| \geq 2^{k-3} d(y)$ (provided that $\delta<1 / 2$ ). This implies

$$
\frac{1}{d-2} \sum_{k \geq 2} \gamma_{k} \int_{A_{k}} \frac{1}{|w-z|^{d-2}} d \mu_{0}(w) \leq \sum_{k \geq 2} \gamma_{k} \mu\left(A_{k}\right)\left(2^{k-3} d(y)\right)^{2-d} \leq 5 \cdot 8^{d-2} .
$$


Note that $\gamma_{1}=1$, which implies that $\mu_{k}=\left(1-\gamma_{k}\right) \mu=\left(1-\gamma_{k}\right) \mu_{0}$. Since $\nu=\sum_{k} \mu_{k}$, we obtain

$$
\begin{gathered}
U^{\nu}(z) \geq \int_{2 \delta d(y)}^{d(y)} \frac{\mu_{x}(B(z, r))}{r^{d-1}} d r+\sum_{k \geq 2} \int_{0}^{\infty} \frac{\mu_{k}(B(z, r))}{r^{d-1}} d r \geq \\
\int_{2 \delta d(y)}^{d(y)} \frac{\mu_{x}(B(x, r-\delta d(y)))}{r^{d-1}} d r+\int_{0}^{\infty} \frac{\mu_{0}(B(z, r))}{r^{d-1}} d r-\frac{1}{d-2} \sum_{k \geq 1} \gamma_{k} \int_{A_{k}} \frac{d \mu_{0}(w)}{|w-z|^{d-2}} \geq \\
\int_{2 \delta d(y)}^{d(y)} c \cdot\left(\frac{r-\delta d(y)}{r}\right)^{d-2} \frac{d r}{r}+\int_{0}^{\infty} \frac{\mu_{0}(B(z, r))}{r^{d-1}} d r-5 \cdot 8^{d-2} \geq c \cdot 2^{2-d} \log \frac{1}{2 \delta}+U^{\mu_{0}}(z)-5 \cdot 8^{d-2} .
\end{gathered}
$$

which implies (28).

To obtain (29), note that for any point $w \in[y, z]$, for $d>2$ we have, by the estimate (12)

$$
\begin{aligned}
& \left|\nabla U^{\mu_{0}}(w)\right| \leq \frac{1}{d-2} \int\left|\nabla \frac{1}{|\xi-w|^{d-2}}\right| d \mu_{0}(\xi)=\int \frac{1}{|\xi-w|^{d-1}} d \mu_{0}(\xi)= \\
& \quad(d-1) \int_{0}^{\infty} \frac{\mu_{0}(B(w, r))}{r^{d}} d r=(d-1) \int_{d(y)}^{\infty} \frac{\mu_{0}(B(w, r))}{r^{d}} d r \leq(d-1) \int_{d(y)}^{\infty} \frac{r^{d-2}}{r^{d}} d r \leq \frac{A}{d(y)}
\end{aligned}
$$

for some constant $A$ depending only on $d$ and $c$. This implies that

$$
U^{\mu_{0}}(z)-U^{\mu_{0}}(y)=\int_{[y, z]} \nabla U^{\mu_{0}}(w) \cdot d w \geq-|z-y| \frac{A}{(1-\delta) d(y)} \geq-A,
$$

which is exactly the equation (29).

Another application of the amalgamation construction will establish the lower bounds required in the case $\alpha<2$.

Lemma 8 (Section 2.3): For any $\alpha<2$ and $c>0$, there exist two constants $\delta$ and $\eta$, such that the following holds.

Let $\Omega$ be an $\alpha$-thick domain in $\mathbb{R}^{d}$ with thickness $c$. Let $y \in \Omega$ and $x \in \partial \Omega$ be the closest point to $y$. Let $\mu \in \mathcal{M}$.

Then either

$$
U(z)>U_{\alpha}^{\mu}(z)+1 \text { whenever }|z-x|<\delta \cdot d(y) \text { and } d(z)>\delta / 4 \cdot d(y) .
$$

or

$$
\mu(B(y, 2 d(y))) \geq \eta d(y)^{d-\alpha}
$$

Proof. Let $\eta=\delta^{d-\alpha+1}$ Assume that $\mu(B(y, 2 d(y)))<\eta d(y)^{d-\alpha}$. Let $\mu_{0}$ be the restriction of $\mu$ to $B(0,2) \backslash B(y, 2 d(y))$, as in the previous lemma. We have, by (10),

$$
\begin{aligned}
U_{\alpha}^{\mu_{0}}(z) \geq U_{\alpha}^{\mu}(z)-\int_{d(z)}^{\infty} \frac{\mu(B(y, 2 d(y)))}{r^{d-\alpha+1}} d r- & \int_{4 d(y)}^{\infty} \frac{\mu(B(y, 4 d(y)))}{r^{d-\alpha+1}} d r \geq \\
& U_{\alpha}^{\mu}(z)-\frac{\eta d(y)^{d-\alpha}}{(d-\alpha) d(z)^{d-\alpha}} \geq U_{\alpha}^{\mu}(z)-\frac{4^{d-\alpha} \delta}{(d-\alpha)} .
\end{aligned}
$$

On the other hand, the same reasoning as the proof of (28) above, gives

$$
U(z) \geq U_{\alpha}^{\nu}(z) \geq U_{\alpha}^{\mu_{0}}(z)-C_{1}+c \cdot 2^{\alpha-d} \log \frac{1}{\delta}
$$

for some constant $C_{1}$ depending only on $d, \alpha$, and $c$. Here, as in (28), $\nu$ is the amalgamation of $\mu$ at $y$. 
Estimates (34) and (35) together imply the statement of the lemma.

3.2. The boundary drift of the WoS process: $\alpha \leq 2$. First we establish that the process $U_{t}$ has the drift toward the boundary in the case $\alpha<2$.

Lemma 9 (Section 2.3): There are constants $L$ and $k$, depending only on $c, \beta$, and $\alpha$, such that

$$
\mathbf{E}\left[\left(U_{t+k}-U_{t}\right) \mid U_{t}\right]>L \text {. }
$$

Proof. Let us fix $X_{t}$. By weak-*-compactness of the set $\mathcal{M}$, there exists a measure $\mu$ such that $U_{\alpha}^{\mu}\left(X_{t}\right)=U\left(X_{t}\right)=U_{t}$. By Lemma 8, there exist $\eta$ and $\delta$, such that either

$$
U(z)>U_{\alpha}^{\mu}(z)+1 \text { whenever }|z-x|<\delta \cdot d\left(X_{t}\right) \text { and } \delta / 4 \cdot d\left(X_{t}\right)<d(z) .
$$

where $x$ is the closest to $X_{t}$ point on $\partial \Omega$, or

$$
\mu\left(B\left(X_{t}, 2 d\left(X_{t}\right)\right)\right) \geq \eta d\left(X_{t}\right)^{d-\alpha}
$$

Let us start with the first case. Pick a number $k$ so that $(1-\beta / 3)^{k}<\delta$. Let us introduce a new stopping time $k(\delta):=\min \left(T_{\delta d\left(X_{t}\right)}-t, k\right)$. Note that by the construction of the WoS algorithm, $d\left(X_{t+k(\delta)}\right)>\delta / 4 \cdot d\left(X_{t}\right)$. Note also that since $k(\delta) \leq k$ and

$$
2^{-k(\delta)} d\left(X_{t}\right) \leq d\left(X_{t+k(\delta)}\right) \leq(1-\beta / 2)^{k(\delta)} d\left(X_{t}\right),
$$

for some $p=p(d, \beta)>0$ we have

$$
\mathbf{P}\left[\left|X_{t+k(\delta)}-x\right|<\delta \cdot d\left(X_{t}\right)\right]>p^{k} .
$$

Let us now observe that by subharmonicity of the functions $U$ and $U_{\alpha}^{\mu}$, the previous estimate, the fact that $U \geq U_{\alpha}^{\mu}$, and the assumption (36),

$$
\begin{aligned}
& \mathbf{E}\left[\left(U_{t+k}-U_{t}\right) \mid X_{t}\right] \geq \mathbf{E}\left[\left(U_{t+k(\delta)}-U_{t}\right) \mid X_{t}\right] \geq \\
& \quad \mathbf{E}\left[\left(U_{\alpha}^{\mu}\left(X_{t+k(\delta)}\right)-U_{\alpha}^{\mu}\left(X_{t}\right)\right) \mid X_{t}\right]+\mathbf{E}\left[U\left(X_{t+k(\delta)}\right)-U_{\alpha}^{\mu}\left(X_{t+k(\delta)}\right) \mid X_{t}\right] \geq \\
& \mathbf{E}\left[U\left(X_{t+k(\delta)}\right)-U_{\alpha}^{\mu}\left(X_{t+k(\delta)}\right) \chi_{\left|X_{t+k(\delta)}-x\right|<\delta \cdot d\left(X_{t}\right)} \mid X_{t}\right] \geq \mathbf{P}\left[\left|X_{t+k(\delta)}-x\right|<\delta \cdot d\left(X_{t}\right)\right]>p^{k} .
\end{aligned}
$$

Since the value of $X_{t}$ determines the value of $U_{t}$, this establishes the statement of Lemma in the first case (with $L=p^{k}$ ).

Now let us consider the second case. By the Green formula, for a $C^{2}$-smooth function $u$,

$$
\mathbf{E}\left[u\left(X_{t+1}\right) \mid X_{t}\right]-u\left(X_{t}\right)=\int_{\beta d\left(X_{t}\right) S^{d}} u(y) d S(y)-u\left(X_{t}\right)=\int_{0}^{\beta d\left(X_{t}\right)} r^{1-d} \int_{B\left(X_{t}, r\right)} \Delta u(y) d V(y) d r
$$

where $S^{d}$ is the unit sphere in $\mathbb{R}^{d}$ with the normalized Lebesgue measure $S$, and $d V$ is the volume element in $\mathbb{R}^{d}$.

Note that by (10) and (9), for $\left|y-X_{t}\right| \leq \beta d\left(X_{t}\right)$ we have

$$
\begin{gathered}
\Delta U_{\alpha}^{\mu}(y)=(d-\alpha+2)(2-\alpha) U_{\alpha-2}^{\mu}(y)= \\
(d-\alpha+2)(2-\alpha) \int_{0}^{\infty} \frac{\mu(B(y, r))}{r^{d-\alpha+3}} d r \geq \\
(d-\alpha+2)(2-\alpha) \mu\left(B\left(X_{t}, 2 d\left(X_{t}\right)\right)\right) \int_{(2+\beta) d\left(X_{t}\right)}^{\infty} \frac{1}{r^{d-\alpha+3}} d r \geq \\
\quad(2-\alpha) \frac{\mu\left(B\left(X_{t}, 2 d\left(X_{t}\right)\right)\right)}{\left((2+\beta) d\left(X_{t}\right)\right)^{d-\alpha+2}} \geq C_{1}\left(d\left(X_{t}\right)\right)^{-2}
\end{gathered}
$$

for some constant $C_{1}$ depending only on $d, \alpha$, and $\beta$. 
So, using (40), applied to $u=U^{\mu}$ and (41), we get

$$
\begin{aligned}
\mathbf{E}\left[U_{t+k} \mid X_{t}\right]-U_{t} \geq \mathbf{E}\left[U_{t+1} \mid X_{t}\right]-U_{t} \geq \mathbf{E}\left[U^{\mu}\left(X_{t+1}\right) \mid X_{t}\right] & -U^{\mu}\left(X_{t}\right) \geq \\
& \int_{0}^{\beta d\left(X_{t}\right)} r^{1-d}\left(C_{1}\left(d\left(X_{t}\right)\right)^{-2} r^{d}\right) d r=C_{1}\left(\left(d\left(X_{t}\right)\right)^{2}\right)\left(d\left(X_{t}\right)\right)^{-2} \beta^{2} / 2=L
\end{aligned}
$$

for some constants $C_{2}$ and $L$ depending only on $d, \alpha$, and $\beta$. Since again the value of $X_{t}$ determines the value of $U_{t}$, the Lemma follows.

Let us now turn to the case $\alpha=2$.

Lemma 11 (Section 2.4): Let $\Omega$ be a 2-thick domain in $\mathbb{R}^{d}$. There are constants $k$ and $L$, depending only on the thickness $c$, the jump ratio $\beta$, and the dimension $d$, such that

$$
\mathbf{E}\left[\left(U_{t+k}-U_{t}\right)^{2} \mid U_{t}\right]>L \text {. }
$$

Proof. Fix $X_{t}$. By Lemma 10, there exists a constant $\delta$, dependent only on $d$ and $c$, such that

$$
U(y)>U\left(X_{t}\right)+1 \text { whenever }|y-x|<\delta \cdot d\left(X_{t}\right) .
$$

This implies that $\left\|U_{t+k}-U_{t}\right\|^{2}>1$ whenever $\left|X_{t+k}-x\right|<\delta d\left(X_{t}\right)$. Note that for some $p>0$ dependent only on $d$ and $\beta$,

$$
\mathbf{P}\left[\left|X_{t+k}-x\right|<(1-\beta / 2)^{k} d\left(X_{t}\right)\right]>p^{k} .
$$

Hence, for sufficiently large $k, \mathbf{P}\left[\left|X_{t+k}-x\right|<\delta d\left(X_{t}\right)\right]>p^{k}$, which, in turn, implies the statement of the lemma.

\section{LOWER BOUNDS: EXAMPLES}

In this section we construct examples of $\alpha$-thick domains for which the bounds in Theorem 2 are tight. The main idea of the construction is as follows.

First of all, we fix $\varepsilon>0$. The thickness constant $C$ is independent of $\varepsilon$ and will be chosen later. We will construct an $\alpha$-thick domain $\Omega_{\alpha, \varepsilon}$ with the thickness $C$. To achieve this we take a domain $A$ to be the unit ball in $\mathbb{R}^{d}$. We remove a "thin" subset of points $D$ from $A$ to obtain $\Omega=A \backslash D$. The set $D$ can be thought of as the subset of the grid $(\gamma \mathbb{Z})^{d}$, for some small $\gamma>0$. The set $D$ will be chosen so that it "separates" the origin from the boundary of $A$. We choose $\gamma$ so that the probability of the WoS originated at 0 hitting a $\varepsilon$-neighborhood of $D$ before hitting the boundary of $A$ is $<1 / 2$ (this means that $D$ is "thin"). Hence, with high probability, the WoS will reach $\partial A$ before terminating. However, in this case the WoS will have to "pass through" the set $D$, where its step magnitudes are bounded by $\gamma$. This will, in turn, yield an $\Omega\left(1 / \gamma^{2}\right)$ bound on the convergence time. The analysis is more intricate in the case when $\alpha=2$. In the case when $\alpha>2$ is not an integer, a slight modification to this construction is needed, as will be described below.

Altogether, in Sections 4.1-4.2 we prove the following:

Claim 15. Let $\alpha \geq 2$ and let $f_{\alpha}(1 / \varepsilon)$ be the growth function given by equation (3) in Theorem 2. Then there is a family of domains $\Omega_{\alpha, \varepsilon} \in \mathbb{R}^{d}$ of constant size, such that $\Omega_{\alpha, \varepsilon}$ is $\alpha$-thick with some constant thickness $C$, and the WoS started at the origin takes an expected $\Omega\left(f_{\alpha}(1 / \varepsilon)\right)$ steps to get $\varepsilon$-close to the boundary.

Proof. (of the lower bound part of Theorem 2 assuming Claim 15).

The lower bound is trivial for $\alpha<2$, since the distance may only decrease by a factor of 2 at each step. 
Our domain $\Omega_{\alpha} \subset \mathbb{R}^{d}$ will be a gluing of "scaled-down" versions of $\Omega_{\alpha, n^{2} \cdot \varepsilon_{n}}$ for an appropriate choice of the sequence $\left\{\varepsilon_{n}\right\}$. The copies will be connected together by $d$-dimensional "tubes". Thus if each $\Omega_{\alpha, n^{2} \varepsilon_{n}}$ (and hence its scaling) is $\alpha$-thick with thickness $C$, the resulting domain $\Omega_{\alpha}$ is $\alpha$-thick with some thickness $C^{\prime}$.

The copy $\Omega_{\alpha, n^{2} \cdot \varepsilon_{n}}$ will be scaled-down by a factor of $n^{2}$ so that the resulting domain $\Omega_{\alpha}$ is bounded. Denote by $x_{n}$ the center of $\Omega_{\alpha, n^{2} \cdot \varepsilon_{n}}$. By Claim 15 we know that the WoS takes at least $\delta \cdot f_{\alpha}\left(1 /\left(n^{2} \varepsilon_{n}\right)\right)$ steps to converge within a distance of $\varepsilon_{n}$ from the boundary.

We have $\delta \cdot f_{\alpha}\left(1 /\left(n^{2} \varepsilon\right)\right) \geq \delta \cdot f_{\alpha}(\varepsilon) / n^{4}$ for all $\alpha$, and $g(1 / \varepsilon)=o(f(1 / \varepsilon))$. Hence for every $n$ there is a sufficiently small $\varepsilon_{n}$ such that $\delta \cdot f_{\alpha}\left(1 /\left(n^{2} \varepsilon\right)\right) \geq g\left(1 / \varepsilon_{n}\right)$. Taking such a sequence $\left\{\varepsilon_{n}\right\}$ completes the proof.

Remark 16. In the lower bound part of Theorem 2 we have shown that there is a domain $\Omega_{\alpha}$ and a sequence of pairs $\left\{x_{n}, \varepsilon_{n}\right\}$ such that $\varepsilon_{n} \searrow 0$ and the WoS from $x_{n}$ takes more than $g\left(1 / \varepsilon_{n}\right)$ to converge within an $\varepsilon_{n}$ distance from the boundary. With a little more work, it is possible to give an example where there is a single $x_{0}$ and a sequence $\left\{\varepsilon_{n}\right\}$ such that $\varepsilon_{n} \searrow 0$ and the WoS from $x_{0}$ takes more than $g\left(1 / \varepsilon_{n}\right)$ to converge within an $\varepsilon_{n}$ distance from the boundary.

4.1. Proof of the lower bound in the case $\alpha>2$. In this section we will give an example of a "thin" $\alpha$-thick domain $\Omega_{\alpha}$ for which the WoS will likely take $\Omega\left(n^{2-4 / \alpha}\right)$ steps to converge within $\varepsilon=1 / n$ from the boundary $\partial \Omega_{\alpha}$, thus proving Claim 15 for $\alpha>2$. The domain $\Omega_{\alpha}$ will reside in $\mathbb{R}^{d}$, where $d=\lceil\alpha\rceil \geq 3$. It is easy to see that the examples in higher dimensions $d^{\prime}>d$ can be constructed from $\Omega_{\alpha}$ by simply multiplying $\Omega_{\alpha}$ by $[-1,1]^{d^{\prime}-d}$, as discussed in Remark 19 below.

The set

$$
\Omega_{\alpha}:=B(0,1)_{d} \backslash S
$$

is comprised of a $d$-dimensional unit ball in $\mathbb{R}^{d}$ with a set of points $S$ removed. We take $A$ to be the "middle $1 / 3$ " shell of the $d$-dimensional ball:

$$
A=\left\{z \in \mathbb{R}^{d}: 1 / 3<|z|<2 / 3\right\} .
$$

Let $0<\gamma \ll 1$ be the grid size that will be selected later. We consider the set $A_{\gamma}$ of gridpoints in A.

$$
A_{\gamma}=(\gamma \mathbb{Z})^{d} \cap A .
$$

Let $0 \leq \eta:=d-\alpha<1$. Denote by $C_{\eta}$ the $\eta$-dimensional Cantor set in the interval $[0,1]$. It is obtained by removing the middle $\lambda$-fraction of the interval, then removing the middle $\lambda$-fraction of each subinterval etc. For the set $C_{\eta}$ to be $\eta$-dimensional, we choose $\lambda$ so that

$$
\eta=\frac{\log 2}{\log 2-\log (1-\lambda)}
$$

In the special case when $\eta=0$, we set $C_{0}=\{0\}$. Note that by Theorem 9.3. from [Fal03], $H^{\eta}\left(C_{\eta}\right)>0$. We can view $C_{\eta}$ as a subset of $\mathbb{R}^{d}$. By self-similarity, the set $C_{\eta}^{c}$ is $(d-\eta)$-thick. Now we define the set $S$ :

$$
S:=A_{\gamma}+\gamma C_{\eta}
$$

In other words, $S$ is obtained by attaching a $\gamma$-scaled copy of $C_{\eta}$ to each gridpoint of $A_{\gamma}$. This completes the definition of the set $\Omega_{\alpha}=B(0,1)_{d} \backslash S$. Each point in $\partial \Omega_{\alpha}$ has an $\eta$-dimensional Cantor set in $\Omega_{\alpha}^{c}$ attached to it. Since $\alpha=d-\eta$, there is a universal constant $C \geq 1 / 16$ such that for every $\gamma$, the set $\Omega_{\alpha}$ is $\alpha$-thick with thickness $C$.

The following two claims assert that for an appropriately chosen $\gamma$, the WoS originated at the origin $0 \in \mathbb{R}^{d}$ and terminated at the $1 / n$ neighborhood of $\partial \Omega_{\alpha}$ is likely to hit the boundary of the unit ball (as opposed to the neighborhood of $S$ ), and is likely to spend $\Omega\left(n^{2-4 / \alpha}\right)$ steps getting there. 
Claim 17. If $\gamma>16 n^{2 / \alpha-1}$ then a WoS originated at 0 and terminated at the $1 / n$-neighborhood of the boundary $\partial \Omega_{\alpha}$ will hit the boundary of the ball $B(0,1)_{d}$ with probability at least 3/4.

Proof. It is not hard to see that we can choose a finite subset $P$ of points in $S$ such that $|P|<$ $2^{\alpha} \gamma^{-\alpha} \cdot n^{\eta}$, and for every $x$ such that $d(x, S)<1 / n$ there is a $p \in P$ such that $|x-p|<2 / n$. Consider the harmonic function

$$
\Phi(x):=\sum_{y \in P} \frac{1}{|x-y|^{d-2}}>0 .
$$

Since the function $\Phi$ is harmonic, its application to the WoS process $X_{t}$ gives a martingale. Hence if $T$ is the stopping time of the process,

$$
\mathbf{E}\left[\Phi\left(X_{T}\right)\right]=\Phi\left(X_{0}\right)=\Phi(0)<3^{d-2} \cdot|P|<4 \cdot 6^{d-2} \gamma^{-\alpha} \cdot n^{\eta} .
$$

On the other hand, if $d\left(X_{T}, S\right)<1 / n$, then there is a $y \in P$ with $\left|X_{T}-y\right|<2 / n$, and

$$
\Phi\left(X_{T}\right)>=1 /\left|y-X_{T}\right|^{d-2}>(n / 2)^{d-2} .
$$

Hence the probability of $X_{T}$ being near $S$ is bounded by

$$
\frac{\mathbf{E}\left[\Phi\left(X_{T}\right)\right]}{(n / 2)^{d-2}}<\frac{4 \cdot 6^{d-2} \gamma^{-\alpha} \cdot n^{\eta}}{(n / 2)^{d-2}}<\frac{16^{d-1} \gamma^{-\alpha}}{4 \cdot n^{\alpha-2}}<\frac{16^{\alpha} n^{2}}{4 \cdot(\gamma n)^{\alpha}}<1 / 4
$$

The last inequality follows from the condition on $\gamma$.

Claim 18. There is a universal constant $\delta>0$ such that for $\gamma$ as above, with probability at least $1 / 2$ the WoS takes at least $\delta(1 / \gamma)^{2}$ steps to reach the boundary of the ball $B(0,1)_{d}$.

Proof. The proof is done analogously to the proof of Claim 22 below.

Hence the expected number of steps is at least

$$
\frac{\delta}{2} \cdot\left(\frac{1}{16 n^{2 / \alpha-1}}\right)^{2}=\Omega\left(n^{2-4 / \alpha}\right),
$$

which completes the proof of the lower bound for Theorem 2 in the case when $\alpha>2$.

Remark 19. To generalize the example from $\mathbb{R}^{d}$ to $\mathbb{R}^{d^{\prime}}$ where $d^{\prime}>d$ we take the domain $\Omega_{\alpha}^{\prime}=\Omega_{\alpha} \times$ $[-1,1]^{d^{\prime}-d}$. Clearly this domain has the same thickness properties as $\Omega_{\alpha}$. To see that Claim 18 still holds, observe that with a constant probability the WoS will approach $\partial B(0,1)_{d} \times(-1,1)^{d^{\prime}-d}$ before "escaping" through the new boundary $\left.B(0,1)_{d} \times \partial[-1,1]\right]^{d^{\prime}-d}$. Conditioned on the WoS approaching $\partial B(0,1)_{d} \times(-1,1)^{d^{\prime}-d}$ first, Claim 18 still holds, giving the desired lower bound.

4.2. Proof of the lower bound in the case $\alpha=2$. We will now give an example of a two dimensional domain $\Omega$ such that the expected convergence time of the WoS to a $O(1 / n)$-neighborhood of $\partial \Omega$ is $\Omega\left(\log ^{2} n\right)$. As in the previous section, by taking the $d$-dimensional domain $\Omega_{d}=\Omega \times[-1,1]^{d-2}$ for $d>2$, we obtain a lower bound of $\Omega\left(\log ^{2} n\right)$ for 2 -thick domains in $\mathbb{R}^{d}$, proving the lower bound for $\alpha=2$ in Theorem 2 .

The domain $\Omega$ will consist of the unit disc in $\mathbb{R}^{2}$ with $O(\log n)$ holes "poked" out of it in a grid formation. More specifically, let $\gamma=4 / \log ^{1 / 2} n$. We consider the grid $\Gamma=\gamma \mathbb{Z} \times \gamma \mathbb{Z} \subset \mathbb{R}^{2}$. We take $\Omega$ to be the unit disc with points from $\Gamma$ removed from the "middle third" annulus of the disc.

$$
\Omega=B(0,1) \backslash((B(0,2 / 3) \backslash B(0,1 / 3)) \cap \Gamma) .
$$

The set $\Omega$ is illustrated on Fig. 4(a).

We will show that a WoS originated at the origin $X_{0}=0$ would require an expected time of $\Omega\left(\log ^{2} n\right)$ to converge. We first observe the following: 
Claim 20. With probability at least $7 / 8, a$ WoS originated at $X_{0}=0$ that runs until $d\left(X_{t}\right)<1 / n$ terminates near the unit circle (and not near one of the holes).

Proof. Let $\left\{a_{i}\right\}_{i=1}^{k}=B(0,1) \backslash \Omega$ be the set of holes in $\Omega$. Define the harmonic function

$$
\Phi(z)=\sum_{i=1}^{k} \log \left(2 /\left|z-a_{i}\right|\right) .
$$

It is clear the $\Phi(z)>0$ for all $z \in B(0,1)$. For any point $u$ in the $1 / n$-neighborhood of any of the holes, $\Phi(u)>\log n$. On the other hand,

$$
\Phi(0)<k \cdot \log 6<2 / \gamma^{2}=(\log n) / 8 .
$$

If $X_{t}$ is the WoS process with $X_{0}=0$ terminated at time $T$ when $d\left(X_{T}, \partial \Omega\right)<1 / n$, then $\Phi\left(X_{t}\right)$ is a martingale. Hence,

$$
(\log n) / 8>\Phi\left(X_{0}\right)=\mathbf{E}\left[\Phi\left(X_{t}\right)\right]>\mathbf{P}\left[X_{t} \text { near a hole }\right] \cdot \log n .
$$

Hence the probability that the WoS terminates near a hole is less than $1 / 8$.

For simplicity, we will assume that at every step for the process the WoS jumps exactly half way to the boundary $\partial \Omega$.

To facilitate the analysis we replace the WoS process $X_{t}$ on $\Omega$ with the following process $Y_{t}$. It evolves in exactly the same fashion as $X_{t}$, except when $Y_{t}$ is closer than $1 / n$ to one of the holes in $\Omega$. In this case, instead of terminating, the process makes a jump of $1 / n$ in a direction selected uniformly at random. The process $Y_{t}$ is guaranteed to terminate near the unit circle. We denote the termination time by $T$. Further, we set $Y_{t}=Y_{T}$ for $t>T$. Note that if the process $X_{t}$ does not terminate near one of the holes, then the process $Y_{t}$ coincides with $X_{t}$. Claim 20 implies that this happens with probability at least $7 / 8$ :

Claim 21. $\mathbf{P}\left[X_{t}\right.$ does not coincide with $\left.Y_{t}\right]<1 / 8$.

We define two regions $A$ and $B, B \subset A \subset \Omega$. We take $A$ to be the union of discs with radius $r=\gamma / 4$ around the holes in $\Omega$. We take $B$ to be the union of discs with radius $4 r / 7$ around the same holes. The sets $\Omega, A$ and $B$ are illustrated on Fig. 4(a).

Let time $t_{0}$ be the first time with $\left|Y_{t}\right|>1 / 2$. Let $t^{\prime}$ be the first time afterwards with either $\left|Y_{t}\right|>2 / 3$ or $\left|Y_{t}\right|<1 / 3$. Our goal is to show that with probability at least $3 / 4,\left|t_{0}-t^{\prime}\right|=\Omega\left(\log ^{2} n\right)$. We define a subprocess $Z_{t}$ of $Y_{t}$ as follows. Let $\left\{s_{i}\right\}_{i=0}^{k}$ be a subsequence of times $s$ between $t_{0}$ and $t^{\prime}$ such that $Y_{s} \notin A$. We set $Z_{i}=Y_{s_{i}}$. We further define $\Delta_{i}=Z_{i}-Z_{i-1}$. An instance of the process $Z_{i}$ is illustrated on Fig. 4(b). Since $Y_{t}$ is a martingale, and $Z_{i}$ is defined by a stopping rule on $Y_{t}$, $Z_{i}$ is also a martingale, i.e.

$$
\mathbf{E}\left[\Delta_{i} \mid \Delta_{1}, \Delta_{2}, \ldots, \Delta_{i-1}\right]=0
$$

In addition, it is not hard to see from the definition of $Y_{t}$ that $\left|\Delta_{i}\right|<4 / \log ^{1 / 2} n$ for all $i$. Let $k$ be the number of steps $Z_{i}$. We can extend $Z_{t}=Z_{k}$ for $t>k$ while preserving the martingale property. Our first claim is that $k=\Omega(\log n)$.

Claim 22. $\mathbf{P}\left[k<10^{-4} \log n\right]<1 / 8$. 


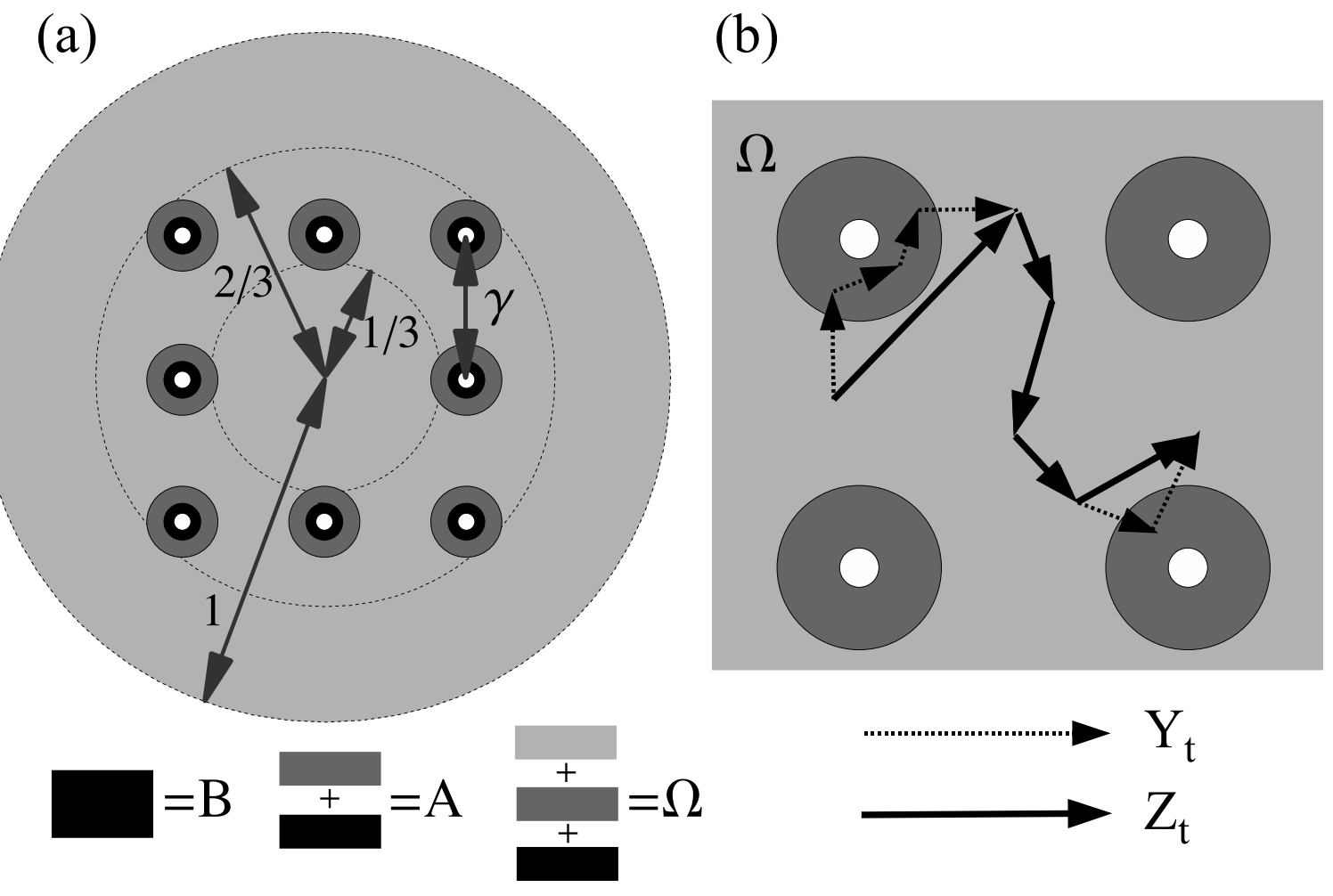

Figure 4. An illustration the sets $\Omega, A$ and $B$ (a), and a possible sequence of jumps in the processes $\left\{Y_{t}\right\}$ and $\left\{Z_{t}\right\}$ (b)

Proof. Denote $\ell=\left\lceil 10^{-4} \log n\right\rceil$. Then, by (46),

$$
\begin{gathered}
\mathbf{E}\left[\left(Z_{0}-Z_{\ell}\right)^{2}\right]=\mathbf{E}\left[\left(\Delta_{1}+\Delta_{2}+\ldots+\Delta_{\ell}\right)^{2}\right]=\sum_{j=1}^{\ell} \mathbf{E}\left[\Delta_{j}^{2}\right]+\sum_{1 \leq i<j \leq \ell} \mathbf{E}\left[\Delta_{i} \Delta_{j}\right]= \\
\sum_{j=1}^{\ell} \mathbf{E}\left[\Delta_{j}^{2}\right]+\sum_{1 \leq i<j \leq \ell} \mathbf{E}\left[\Delta_{i} \cdot \mathbf{E}\left[\Delta_{j} \mid \Delta_{i}\right]\right]=\sum_{j=1}^{\ell} \mathbf{E}\left[\Delta_{j}^{2}\right]<\ell \cdot 16 / \log n<1 / 288 .
\end{gathered}
$$

On the other hand, by definition, $\left|Z_{0}-Z_{k}\right|>1 / 6$, and $\left(Z_{0}-Z_{k}\right)^{2}>1 / 36$. Thus,

$$
\mathbf{P}[k \leq \ell] \leq \mathbf{P}\left[Z_{\ell}=Z_{k}\right]<(1 / 288) /(1 / 36)=1 / 8 .
$$

Thus the number of steps the process $Z_{t}$ takes is at least $10^{-4} \log n$ w.p. $>7 / 8$. The process $Y_{t}$ consists of the steps of the process $Z_{t}$ plus, in addition, steps the process takes within the region $A$. We claim that once the process $Y_{t}$ enters the region $A$, it is expected to spend $\Omega(\log n)$ steps there. Moreover, the following holds.

Claim 23. Let $\eta>2$. Then there is a $\theta>0$ such that whenever $Y_{t} \in A$, if $s>t$ is the first time, conditioned on $Y_{t}$ such that $Y_{s} \notin A$, then

$$
\mathbf{P}\left[s-t>\theta \log ^{2} n\right]>\eta / \log n,
$$

for sufficiently large $n$. 
Proof. Denote the hole in $\Omega$ that is closest to $Y_{t}$ by $x$. Given that $Y_{t} \in A$, there is some fixed probability $p>0$ that $Y_{t+1} \in B$. In other words, $\left|Y_{t+1}-x\right|<4 r / 7=\gamma / 7$. Consider the harmonic function

$$
\Phi(z)=\log (r /|x-z|) .
$$

Let $t^{\prime}>t+1$ be the first time such that either $Y_{t^{\prime}} \notin A$ (and thus $t^{\prime}=s$ ), or $\left|Y_{t^{\prime}}-x\right|<n^{-p /(5 \eta)}$. If $Y_{t^{\prime}} \notin A$, then $\Phi\left(Y_{t^{\prime}}\right)<0$. In the other case, $\Phi\left(Y_{t^{\prime}}\right)<p \log n /(4 \eta)$. Since $t^{\prime}$ is a stopping time, the optional stopping time theorem applied to the martingale $\Phi\left(Y_{t+\tau}\right)$ combined with the estimate $\Phi\left(Y_{t}\right)>1 / 2$, gives

$$
\mathbf{P}\left[\left|Y_{t^{\prime}}-x\right|<n^{-p /(5 \eta)}\right]>\frac{1 / 2}{p \log n /(4 \eta)}=2 \eta /(p \log n) .
$$

To complete the argument, we claim that assuming $\left|Y_{t^{\prime}}-x\right|<n^{-p /(5 \eta)}$, it will take the process another $\Omega\left(\log ^{2} n\right)$ steps to escape $A$ with probability at least $1 / 2$. We consider the process $\phi_{\tau}=$ $\Phi\left(Y_{t^{\prime}+\tau}\right)$ stopped at time $\tau_{0}$ when either $Y_{t^{\prime}+\tau_{0}}$ escapes $A$, or gets closer than distance $1 / n$ from $x$. $\phi_{\tau}$ is a bounded martingale. Moreover, it is not hard to see that $\left|\phi_{0}-\phi_{\tau_{0}}\right|>p \log n /(6 \eta)$, and $\left|\phi_{i}-\phi_{i+1}\right|<1$ for all $i$. These two facts imply that

$$
\mathbf{E}\left[\tau_{0}\right]>\sum_{i=1}^{\tau_{0}} \mathbf{E}\left[\left(\phi_{i}-\phi_{i-1}\right)^{2} \chi_{i<\tau_{0}}\right]=\mathbf{E}\left[\left(\phi_{\tau_{0}}-\phi_{0}\right)^{2}\right]>(p \log n /(6 \eta))^{2}=p^{2} \log ^{2} n /\left(36 \eta^{2}\right) .
$$

Markov's inequality implies that $\theta=p^{2} /\left(72 \eta^{2}\right)$ satisfies the statement of the claim.

By Claim 22 we know that except with probability $<1 / 8$ the walk will contain at least $\Omega(\log n)$ visits to $A$. It remains to use Claim 23 to show that at least one of these stays must be $\Omega\left(\log ^{2} n\right)$ long. Recall that $T$ is the stopping time of the process $Y_{T}$, and $k$ is the number of steps $Y_{t}$ takes outside of $A$.

Claim 24. Let $\alpha_{1}=10^{-4}$ from Claim 22. There is a constant $\alpha_{2}>0$ such that

$$
\mathbf{P}\left[k>\alpha_{1} \log n \text { and } T<\alpha_{2} \log ^{2} n\right]<1 / 8 .
$$

Proof. For every $t$ such that $1 / 3<\left|Y_{t}\right|<2 / 3$ and $Y_{t} \notin A$, there is a probability $p_{1}>0$ such that either $Y_{t+1} \in A$ or $Y_{t+2} \in A$. By Claim 23 we can choose $\alpha_{2}>0$ such that whenever $Y_{t^{\prime}} \in A$, the process $Y_{t^{\prime}+\tau}$ does not escape $A$ for at least $\alpha_{2} \log ^{2} n$ with probability at least $p_{2}=6 /\left(\alpha_{1} p_{1} \log n\right)$. Hence for each $1 / 3<\left|Y_{t}\right|<2 / 3$ with $Y_{t} \notin A$, the probability that $Y_{t+1}$ or $Y_{t+2}$ enters $A$, and stays there for at least $\alpha_{2} \log ^{2} n$ steps is at least $p_{1} \cdot p_{2}=6 /\left(\alpha_{1} \log n\right)$. Since there are at least $k=\alpha_{1} \log n$ $Y_{t}$ 's satisfying $1 / 3<\left|Y_{t}\right|<2 / 3$ and $Y_{t} \notin A$, the probability that for neither one of them does $Y_{t+1}$ or $Y_{t+2}$ enter $A$, and stay there for at least $\alpha_{2} \log ^{2} n$ steps is at most

$$
\left(1-6 /\left(\alpha_{1} \log n\right)\right)^{k / 2}<\left(1-6 /\left(\alpha_{1} \log n\right)\right)^{\alpha_{1} \log n / 2}<e^{-\left(6 /\left(\alpha_{1} \log n\right)\right) \cdot\left(\alpha_{1} \log n / 2\right)}=e^{-3}<1 / 8 .
$$

Claims 21, 22 and 24 imply the following.

Claim 25. Let $X_{t}$ be the WoS process on the set $\Omega$ with $X_{0}=0$. Let $T^{\prime}$ be its termination time. Then

$$
\mathbf{P}\left[T^{\prime}>\alpha_{2} \log ^{2} n\right]>5 / 8,
$$

where $\alpha_{2}>0$ is the constant from Claim 24. In particular, this implies that $\mathbf{E}\left[T^{\prime}\right]=\Omega\left(\log ^{2} n\right)$.

Proof. We know that $T^{\prime}>\alpha_{2} \log ^{2} n$ if the following three conditions hold: (C1) the process $X_{t}$ coincides with the process $Y_{t} ;(\mathrm{C} 2)$ the process $Y_{t}$ makes at least $k>\alpha_{1} \log n$ steps outside of $A$ in the $\{z: 1 / 3<|z|<2 / 3\}$ annulus; and (C3) the stopping time $T$ of $Y_{t}$ satisfies $T>\alpha_{2} \log ^{2} n$. In 
fact conditions (C1) and (C3) suffice. We have $\mathbf{P}[\overline{C 1}]<1 / 8$ by Claim $21, \mathbf{P}[\overline{C 2}]<1 / 8$ by Claim 22 , and $\mathbf{P}[C 2 \cap \overline{C 3}]<1 / 8$ by Claim 24 . Here $\bar{C}$ denotes the complement of an event $C$. Hence

$$
\mathbf{P}[\overline{C 1} \cup \overline{C 2} \cup \overline{C 3}] \leq \mathbf{P}[\overline{C 1}]+\mathbf{P}[\overline{C 2}]+\mathbf{P}[C 2 \cap \overline{C 3}]<3 / 8
$$

and

$$
\mathbf{P}\left[T^{\prime}>\alpha_{2} \log ^{2} n\right] \geq \mathbf{P}[C 1 \cap C 2 \cap C 3]=1-\mathbf{P}[\overline{C 1} \cup \overline{C 2} \cup \overline{C 3}]>5 / 8 .
$$

Claim 25 gives the lower bound for Theorem 2 in the case $\alpha=2$.

\section{REFERENCES}

[BB07] I. Binder and M. Braverman. Derandomization of Euclidean Random Walks. LNCS, 4627:353-365, 2007.

[BC06] M. Braverman and S. Cook. Computing over the reals: Foundations for scientific computing. Notices of the AMS, 53(3):318-329, 2006.

[BW99] V. Brattka and K. Weihrauch. Computability of subsets of Euclidean space I: Closed and compact subsets. Theoretical Computer Science, 219:65-93, 1999.

[Car67] Lennart Carleson. Selected problems on exceptional sets. Van Nostrand Mathematical Studies, No. 13. D. Van Nostrand Co., Inc., Princeton, N.J.-Toronto, Ont.-London, 1967.

[EKM97] P. Embrechts, C. Klüppelberg, and T. Mikosch. Modelling Extremal Events for Insurance and Finance. Springer, 1997.

[EKMS80] B. S. Elepov, A. A. Kronberg, G. A. Mihaŭlov, and K. K. Sabel'fel'd. Reshenie kraevykh zadach metodom Monte-Karlo. "Nauka" Sibirsk. Otdel., Novosibirsk, 1980.

[Fal03] Kenneth Falconer. Fractal geometry. John Wiley \& Sons Inc., Hoboken, NJ, second edition, 2003. Mathematical foundations and applications.

[GM04] J. B. Garnett and D. E. Marshall. Harmonic Measure. Cambridge Univ Press, 2004.

[Kak44] Shizuo Kakutani. Two-dimensional Brownian motion and harmonic functions. Proc. Imp. Acad. Tokyo, 20:706-714, 1944.

[KS91] I. Karatzas and S. E. Shreve. Brownian Motion and Stochastic Calculus. Springer Verlag, 2 edition, 1991.

[KS98] I. Karatzas and S.E. Shreve. Methods of Mathematical Finance. Springer, 1998.

[Lan72] N. S. Landkof. Foundations of modern potential theory. Translated from the Russian by AP Doohovskoy, volume 180. 1972.

[Maz02] R.M. Mazo. Brownian Motion: Fluctuations, Dynamics, and Applications. Oxford University Press, 2002.

[Mih79] G. A. Mihallov. Estimation of the difficulty of simulating the process of "random walk on spheres" for some types of regions. Zh. Vychisl. Mat. i Mat. Fiz., 19(2):510-515, 558-559, 1979.

[Mil95] G. N. Milstein. Numerical Integration of Stochastic Differential Equations. Kluwer Academic Publishers, Dodrecht, 1995.

[Mot59] Minoru Motoo. Some evaluations for continuous Monte Carlo method by using Brownian hitting process. Ann. Inst. Statist. Math. Tokyo, 11:49-54, 1959.

[Mul56] M. E. Muller. Some continuous Monte Carlo methods for the Dirichlet problem. Ann. Math. Statist., 27:569-589, 1956.

[Nel67] E. Nelson. Dynamical Theories of Brownian Motion. Princeton University Press Princeton, NJ, 1967.

[Szn98] A.S. Sznitman. Brownian Motion, Obstacles and Random Media. Springer, 1998.

[Wei00] K. Weihrauch. Computable Analysis. Springer-Verlag, Berlin, 2000.

ILIA BINDER, DEPT. OF MATHEMATICS, UNIVERSITY OF TORONTO.

MARK BRAVERMAN, UNIVERSITY OF TORONTO 